\title{
Cytotoxic Cycloartane Triterpenes of the Traditional Chinese Medicine "Shengma" (Cimicifuga dahurica)
}

Authors

Affiliations
Yin Nian ${ }^{1,2}$, Hai-Yan Wang ${ }^{1,2}$, Lin Zhou ${ }^{1}$, Jia Su ${ }^{1,2}$, Yan Li ${ }^{1}$, Ming-Hua Qiu

${ }^{1}$ State Key Laboratory of Phytochemistry and Plant Resources in West China, Kunming Institute of Botany, Chinese Academy of Sciences, Kunming, PR China

${ }^{2}$ Graduate School of the Chinese Academy of Sciences, Beijing, PR China
Key words

- Ranunculaceae

- Cimicifuga dahurica

- cycloartane triterpenes

- cytotoxicity

- structure-activity relationships received June 7,2012

revised October 22, 2012

accepted October 29, 2012

Bibliography

DOI http://dx.doi.org/

10.1055/s-0032-1328019

Published online December 5 ,

2012

Planta Med 2013; 79: 60-69

(C) Georg Thieme Verlag KG

Stuttgart - New York .

ISSN 0032-0943

\section{Correspondence}

Prof. Ming-Hua Qiu

State Key Laboratory of Phyto-

chemistry and Plant Resources

in West China

Kunming Institute of Botany

Graduate School of the Chinese

Academy of Sciences

132 Lanhei Road

Kunming 650204

PR China

Phone: +868715223327

Fax: + 868715223255

mhchiu@mail.kib.ac.cn

\section{Abstract}

$\nabla$

Twelve new 9,19-cycloartane triterpenes (1-12), together with fourteen known compounds (1326), were isolated from the roots of Cimicifuga dahurica. Their structures were determined by application of spectroscopic analyses and chemical methods. Biological evaluation of the compounds against human HL-60, SMMC-7721, A549, MCF-7, and SW-480 cell lines indicated that cimigenoltype glycosides $(\mathbf{1 - 3}, \mathbf{1 9}$, and $\mathbf{2 0})$ showed broadspectrum and moderate cytotoxicities, with $\mathrm{IC}_{50}$

\section{Introduction}

Cancer has become one of the major causes of mortality in humans throughout the world. In 2007, 7.6 million people died from cancer. Significantly, 27 million new cancer cases and 17.5 million cancer deaths are projected to occur in the world by 2050 [1]. Natural products have been a rich source of antitumor agents, and approximately $60 \%$ of currently available drugs are natural compounds or are related to them (from 1940 to 2010) [2].

As one of the three ancient medicinal systems, traditional Chinese medicine (TCM), has gained increasing acceptance and has been recognized by pharmaceutical enterprises as a fountainhead of antitumor drugs [3]. According to the theories of TCM, cancer is caused by imbalances between endogenous physical conditions of the body and exogenous pathogenic factors, including accumulated toxins, heat, and blood stasis [3]. Therefore, the roots of Cimicifuga foetida, belonging to the family Ranunculaceae and officially listed in the Chinese Pharmacopoeia with the name "shengma" as a cooling and detoxicating agent [4], were chosen as the object to investigate potential antitumor constituents. As a result, a number of cytotoxic 9,19-cycloartane triterpenes were succes- values ranging from 4.2 to $14.5 \mu \mathrm{M}$. Meanwhile, cimigenol-type aglycones $(\mathbf{6 - 8}, \mathbf{1 5}, \mathbf{1 6}$, and 18 ) exhibited broad-spectrum and week cytotoxicities, having $\mathrm{IC}_{50}$ values around $20 \mu \mathrm{M}$. In addition, the key points of the structure-activity relationships of aglycones with a cimigenol skeleton were discussed.

Supporting information available online at http://www.thieme-connect.de/ejournals/toc/ plantamedica 
drocimigenol-3-O-[2'-O-acetyl]- $\beta$-D-xylopyranoside (20) [24], 23-epi-26-deoxyactein (21) [25], actein (22) [26], 24-0-acetyl-7 (8)-en-hydroshengmanol (23) [27], 24-O-acetylisodahurinol-3$O$-[2'-O-acetyl]- $\alpha$-L-arabinopyranoside (24) [25], 23-O-acetylshengmanol-3-O- $\beta$-D-xylopyranoside (25) [28], and cimiracemonol B (26) [29], were isolated and identified ( 0 Fig. 1). The isolated compounds were evaluated for their cytotoxicities against human HL-60, SMMC-7721, A549, MCF-7, and SW480 cell lines, using the MTT method. Described herein are the isolation, structure elucidation, and biological activities of the compounds.

\section{Materials and Methods}

$\nabla$

\section{General experimental procedures}

Optical rotations were measured in $\mathrm{MeOH}$ with a Horiba SEAP300 polarimeter. ${ }^{1} \mathrm{H}$ and ${ }^{13} \mathrm{C}$ NMR spectra were recorded in pyridine- $d_{5}$ on Bruker DRX-500 and Avance III-600MHz spectrometers (Bruker). Unless otherwise specified, chemical shifts $(\delta)$ are expressed in ppm with respect to the solvent signals. ESIMS and HR-TOF-ESIMS data were obtained using a VG Autospec-3000 spectrometer. Infrared spectra were recorded on a Shimadzu IR450 instrument with $\mathrm{KBr}$ pellets. Thin-layer chromatography was performed on precoated TLC plates (200-250 $\mu$ m thickness, silica gel $60 \mathrm{~F}_{254}$; Qingdao Marine Chemical, Inc.), and spots were visualized by heating after spraying with $10 \%$ aq. $\mathrm{H}_{2} \mathrm{SO}_{4}$ sol. Semipreparative HPLC was performed on an Agilent 1100 liquid chromatograph with a YMC-Pack Pro C18 RS $10 \mathrm{~mm} \times 250 \mathrm{~mm}$ column. Silica gel (200-300 mesh; Qingdao Marine Chemical, Inc.), Lichroprep RP-18 (40-63 $\mu \mathrm{m}$; Merck), and Sephadex LH-20 (20$150 \mu \mathrm{m}$; Pharmacia) were used for column chromatography (cc).

\section{Plant material}

The roots of Cimicifuga dahurica M. $(0.9 \mathrm{~kg})$ were collected from Qingyuan County, Liaoning Province, China, in September 2006 and identified by Prof. Shengji Pei, Kunming Institute of Botany, Chinese Academy of Sciences. A voucher specimen (KUN No. 200609004) has been deposited at the State Key Laboratory of Phytochemistry and Plant Resources in West China, Kunming Institute of Botany, Chinese Academy of Sciences, PR China.

\section{Extraction and isolation}

The dried and milled roots of Cimicifuga dahurica $(0.9 \mathrm{~kg})$ were extracted with $\mathrm{MeOH}(3 \times 3 \mathrm{~L} \times 24 \mathrm{~h})$ at room temperature to give a residue $(106 \mathrm{~g})$ after evaporating in vacuum at $50^{\circ} \mathrm{C}$. The extract was subjected to silica gel cc $(2 \mathrm{~kg}, 10 \times 150 \mathrm{~cm})$ and eluted with $\mathrm{CHCl}_{3}-\mathrm{MeOH}$ [100:0 (2 L), 50:1 (4L), 20:1 (5 L), 10:1 (4L), $0: 100$ (3 L)] to afford fractions A (21.5 g), B (13.1 g), C (14.5 g), D $(16.8 \mathrm{~g})$, and $\mathbf{E}(16.2 \mathrm{~g})$. Fraction $\mathbf{B}(13.1 \mathrm{~g})$ was divided into five sub-fractions (B.1-B.5) after performing RP-18 cc (180 g, $5 \times 25 \mathrm{~cm}$ ), eluting with $\mathrm{MeOH}-\mathrm{H}_{2} \mathrm{O}$ (gradient from $60: 40$ to $100: 0,10 \mathrm{~L})$. Fraction B.3 (1.5 g) was subjected to repeated silica gel cc $(40 \mathrm{~g}, 4 \times 40 \mathrm{~cm})$ eluted with $\mathrm{CHCl}_{3}-\mathrm{Me}_{2} \mathrm{CO}$ (gradient from $20: 1$ to $10: 1,4 \mathrm{~L}$ ) and then to repeated semipreparative HPLC (eluted with $\mathrm{CH}_{3} \mathrm{CN}-\mathrm{H}_{2} \mathrm{O}$, gradient from $60: 40$ to $85: 15$ ) to yield 4 (3.0 mg), 5 (3.0 mg), 6 (2.0 mg), 13 (4.0 mg), 16 (4.7 mg), and 26 (3.6 mg). Compounds 7 (2.8 mg), 14 (3.2 mg), 15 (3.5 mg), 17 (2.3 mg), $\mathbf{1 8}(2.5 \mathrm{mg})$, and $\mathbf{2 3}(2.2 \mathrm{mg})$ were purified from fraction B.4 $(1.8 \mathrm{~g})$ by silica gel cc $(30 \mathrm{~g}, 3.5 \times 40 \mathrm{~cm})$ eluted with $\mathrm{CHCl}_{3}{ }^{-}$ $\mathrm{Me}_{2} \mathrm{CO}(20: 1,3 \mathrm{~L})$, followed by repeated semipreparative HPLC (eluted with $\mathrm{CH}_{3} \mathrm{CN}-\mathrm{H}_{2} \mathrm{O}$, gradient from $65: 35$ to $85: 15$ ). Fraction B.5 $(0.9 \mathrm{~g})$ was applied to a silica gel column $(30 \mathrm{~g}$,
$3.5 \times 40 \mathrm{~cm})$ eluted with $\mathrm{CHCl}_{3}-\mathrm{Me}_{2} \mathrm{CO}(20: 1,3 \mathrm{~L})$, then purified over semipreparative HPLC (eluted with $\mathrm{CH}_{3} \mathrm{CN}-\mathrm{H}_{2} \mathrm{O}$, gradient from $65: 35$ to $90: 10)$ to afford $8(2.7 \mathrm{mg}), \mathbf{9}(1.2 \mathrm{mg}), 10$ $(1.9 \mathrm{mg})$, and 11 (1.6 mg). Fraction C (14.5 g) was fractionated into four subfractions (C.1-C.4) by performing RP-18 cc (180 g, $5 \times 25 \mathrm{~cm}$ ) eluted with $\mathrm{MeOH}-\mathrm{H}_{2} \mathrm{O}$ (gradient from 60:40 to $90: 10,12 \mathrm{~L})$. Fraction C.3 (2.8 g) was subjected to silica gel cc $\left(50 \mathrm{~g}, 4 \times 40 \mathrm{~cm}\right.$ ) eluted with $\mathrm{CHCl}_{3}-\mathrm{Me}_{2} \mathrm{CO}$ (gradient from 10:1 to $5: 1,4 \mathrm{~L}$ ), then to repeated semipreparative HPLC (eluted with $\mathrm{CH}_{3} \mathrm{CN}-\mathrm{H}_{2} \mathrm{O}$, gradient from $60: 40$ to $\left.75: 25\right)$ to give $\mathbf{1}(5.5 \mathrm{mg}), 2$ (4.8 mg), $\mathbf{3}$ ( $4.3 \mathrm{mg}$ ), $\mathbf{1 2}$ (5.3 mg), 19 (3.3 mg), and $\mathbf{2 4}$ (28 mg). Fractions C.1 and C.2 (4.8 g) were chromatographed on silica gel cc $(50 \mathrm{~g}, 4 \times 40 \mathrm{~cm})$, eluting with $\mathrm{CHCl}_{3}-\mathrm{Me}_{2} \mathrm{CO}(10: 1,8 \mathrm{~L})$ to yield 20 (5.2 mg), 21 (6.0 mg), 22 (5.2 mg), and 25 ( $4.2 \mathrm{mg})$.

Cimigenol-3-O-[2'-O-(E)-2-butenoyl]- $\alpha$-L-arabinopyranoside (1): A white powder; $[\alpha]_{D}^{24}=18.67(c 0.10, \mathrm{MeOH})$; IR $(\mathrm{KBr}): v_{\max }=$ 3452, 2931, 2870, 1727, 1632, 1458, 1383, 1044, $977 \mathrm{~cm}^{-1} ;{ }^{1} \mathrm{H}$ $\left(\mathrm{C}_{5} \mathrm{D}_{5} \mathrm{~N}, 500 \mathrm{MHz}\right)$ and ${ }^{13} \mathrm{C} \mathrm{NMR}\left(\mathrm{C}_{5} \mathrm{D}_{5} \mathrm{~N}, 150 \mathrm{MHz}\right)$ spectra, see O Tables 1 and 2; ESIMS: $m / z=711[\mathrm{M}+\mathrm{Na}]^{+}$; HR-TOF-ESIMS: $m / z=711.4065$ (calc. for $\mathrm{C}_{39} \mathrm{H}_{60} \mathrm{O}_{10} \mathrm{Na}, 711.4084$ ).

25-O-Acetylcimigenol-3-0-[4'-O-acetyl]- $\alpha$-L-arabinopyranoside (2): A white powder; $[\alpha]_{D}^{4}=6.67(c \quad 0.06, \mathrm{MeOH})$; IR $(\mathrm{KBr})$ : $v_{\max }=3472,2968,2870,1739,1458,1374,1072,972 \mathrm{~cm}^{-1} ;{ }^{1} \mathrm{H}$ $\left(\mathrm{C}_{5} \mathrm{D}_{5} \mathrm{~N}, 500 \mathrm{MHz}\right)$ and ${ }^{13} \mathrm{C}$ NMR $\left(\mathrm{C}_{5} \mathrm{D}_{5} \mathrm{~N}, 125 \mathrm{MHz}\right)$ spectra, see - Tables 1 and 2; ESIMS: $m / z=727$ [M + Na] $]^{+}$; HR-TOF-ESIMS: $m / z=727.4029$ (calc. for $\mathrm{C}_{39} \mathrm{H}_{60} \mathrm{O}_{11} \mathrm{Na}, 727.4033$ ).

25-O-Acetylcimigenol-3-O-[3'-O-acetyl]- $\alpha$-L-arabinopyranoside (3): A white powder; $[\alpha]_{\mathrm{D}}^{24}=-6.80(\mathrm{c} 0.05, \mathrm{MeOH})$; IR $(\mathrm{KBr})$ : $v_{\max }=3467,2935,2870,1741,1458,1375,1067,972 \mathrm{~cm}^{-1} ;{ }^{1} \mathrm{H}$ $\left(\mathrm{C}_{5} \mathrm{D}_{5} \mathrm{~N}, 500 \mathrm{MHz}\right)$ and ${ }^{13} \mathrm{C} \mathrm{NMR}\left(\mathrm{C}_{5} \mathrm{D}_{5} \mathrm{~N}, 125 \mathrm{MHz}\right)$ spectra, see - Tables 1 and 2; ESIMS: $m / z=703$ [M - H] ; HR-TOF-ESIMS: $m /$ $z=703.4055$ (calc. for $\mathrm{C}_{39} \mathrm{H}_{59} \mathrm{O}_{11}, 703.4057$ ).

$12 \beta$-Hydroxy-7(8)-en-cimigenol (4): A white powder; $[\alpha]_{D}^{24}=3.20$ (c 0.10, MeOH); IR (KBr): $v_{\max }=3423,2931,2872,1638,1447$, $1381,1025,979 \mathrm{~cm}^{-1} ;{ }^{1} \mathrm{H}\left(\mathrm{C}_{5} \mathrm{D}_{5} \mathrm{~N}, 500 \mathrm{MHz}\right)$ and ${ }^{13} \mathrm{C}$ NMR $\left(\mathrm{C}_{5} \mathrm{D}_{5} \mathrm{~N}, 125 \mathrm{MHz}\right)$ spectra, see 0 Tables 1 and 2; ESIMS: $\mathrm{m} /$ $z=525[\mathrm{M}+\mathrm{Na}]^{+}$; HR-TOF-ESIMS: $m / z=525.3192$ (calc. for $\left.\mathrm{C}_{30} \mathrm{H}_{46} \mathrm{O}_{6} \mathrm{Na}, 525.3192\right)$.

$11 \beta$-Hydroxy-7(8)-en-cimigenol (5): A white powder; $[\alpha]_{D}^{24}=0.47$ (c 0.10, MeOH); IR (KBr): $v_{\max } 3441,2997,2882,1634,1447$, 1380, 1027, $973 \mathrm{~cm}^{-1} ;{ }^{1} \mathrm{H}\left(\mathrm{C}_{5} \mathrm{D}_{5} \mathrm{~N}, 500 \mathrm{MHz}\right)$ and ${ }^{13} \mathrm{C} \mathrm{NMR}$ $\left(\mathrm{C}_{5} \mathrm{D}_{5} \mathrm{~N}, 125 \mathrm{MHz}\right)$ spectra, see 0 Tables 1 and 2; ESIMS: $\mathrm{m} /$ $z=525[\mathrm{M}+\mathrm{Na}]^{+}$; HR-TOF-ESIMS: $\mathrm{m} / z=525.3199$ (calc. for $\left.\mathrm{C}_{30} \mathrm{H}_{46} \mathrm{O}_{6} \mathrm{Na}, 525.3192\right)$.

11 $\beta$-Hydroxy-15-deoxycimigenol-7(8)-en-3-one (6): A white powder; $[\alpha]_{D}^{24}=-23.33(\mathrm{MeOH}, c 0.05)$; IR $(\mathrm{KBr}): v_{\max }=3441$, 2969, 2869, 1711, 1633, 1382, $975 \mathrm{~cm}^{-1} ;{ }^{1} \mathrm{H}\left(\mathrm{C}_{5} \mathrm{D}_{5} \mathrm{~N}, 500 \mathrm{MHz}\right)$ and ${ }^{13} \mathrm{C}$ NMR $\left(\mathrm{C}_{5} \mathrm{D}_{5} \mathrm{~N}, 150 \mathrm{MHz}\right)$ spectra, see 0 Tables 1 and 2; ESIMS: $m / z=507[\mathrm{M}+\mathrm{Na}]^{+}$; HR-TOF-ESIMS: $m / z=507.3090$ (calc. for $\mathrm{C}_{30} \mathrm{H}_{44} \mathrm{O}_{5} \mathrm{Na}$, 507.3086).

Cimigenol-7(8)-en-3-one (7): A white powder; $[\alpha]_{D}^{24}=-21.67(c$ 0.10, MeOH); IR $(\mathrm{KBr}): v_{\text {max }}=3492,2984,2839,1709,1637$, $1452,1382,1024,987 \mathrm{~cm}^{-1} ;{ }^{1} \mathrm{H}\left(\mathrm{C}_{5} \mathrm{D}_{5} \mathrm{~N}, 500 \mathrm{MHz}\right)$ and ${ }^{13} \mathrm{C} \mathrm{NMR}$ $\left(\mathrm{C}_{5} \mathrm{D}_{5} \mathrm{~N}, 125 \mathrm{MHz}\right)$ spectra, see 0 Tables 1 and 2; ESIMS: $\mathrm{m} /$ $z=507[\mathrm{M}+\mathrm{Na}]^{+}$; HR-TOF-ESIMS: $m / z=507.3086$ (calc. for $\mathrm{C}_{30} \mathrm{H}_{44} \mathrm{O}_{5} \mathrm{Na}$, 507.3086).

Cimigenol-1(2),7(8)-dien-3-one (8): A white powder; $[\alpha]_{D}^{24}=$ -60.60 (c 0.10, MeOH); IR (KBr): $v_{\max }=3432,2947,2852,1693$, $1637,1460,1382,1070,978 \mathrm{~cm}^{-1} ;{ }^{1} \mathrm{H}\left(\mathrm{C}_{5} \mathrm{D}_{5} \mathrm{~N}, 500 \mathrm{MHz}\right)$ and ${ }^{13} \mathrm{C}$ NMR $\left(\mathrm{C}_{5} \mathrm{D}_{5} \mathrm{~N}, 125 \mathrm{MHz}\right)$ spectra, see 0 Tables 1 and 2; ESIMS: $\mathrm{m} /$ $z=505[\mathrm{M}+\mathrm{Na}]^{+}$; HR-TOF-ESIMS: $m / z=505.2944$ (calc. for $\mathrm{C}_{30} \mathrm{H}_{42} \mathrm{O}_{5} \mathrm{Na}$, 505.2929). 


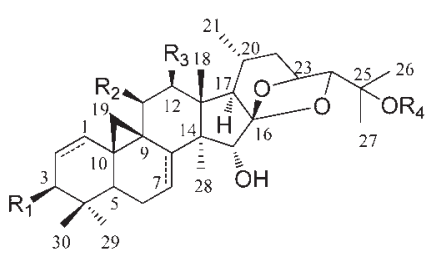

$1 \mathrm{R}_{\mathbf{1}}=O-2^{\prime}-O-(E)$-2-butenoyl- $\alpha$-L-arabinose, $\mathrm{R}_{2}=\mathrm{H}, \mathrm{R}_{3}=\mathrm{H}, \mathrm{R}_{4}=\mathrm{H}, 24 \mathrm{~S}$ $2 \mathrm{R}_{1}=0-4^{\prime}-O$-acetyl- $\alpha$-L-arabinose, $\mathrm{R}_{2}=\mathrm{H}, \mathrm{R}_{3}=\mathrm{H}, \mathrm{R}_{4}=\mathrm{Ac}, 24 \mathrm{~S}$

$3 \mathrm{R}_{1}=O-3^{\prime}-O$-acctyl- $\alpha$-L-arabinose, $\mathrm{R}_{2}=\mathrm{H}, \mathrm{R}_{3}=\mathrm{H}, \mathrm{R}_{4}=\mathrm{Ac}, 24 \mathrm{~S}$

$4 \mathrm{R}_{1}=\mathrm{OII}, \mathrm{R}_{2}=\mathrm{H}, \mathrm{R}_{3}=\mathrm{OH}, \mathrm{R}_{4}=\mathrm{H}, \Delta 7(8), 24 \mathrm{~S}$

$5 \mathrm{R}_{1}=\mathrm{OH}, \mathrm{R}_{2}=\mathrm{OH}, \mathrm{R}_{3}=\mathrm{H}, \mathrm{R}_{4}=\mathrm{H}, \Delta 7(8), 24 \mathrm{~S}$

$7 \mathrm{R}_{\mathbf{1}}==\mathrm{O}, \mathrm{R}_{7}=\mathrm{H}, \mathrm{R}_{3}=\mathrm{H}, \mathrm{R}_{4}=\mathrm{H}, \triangle 7(8), 24 \mathrm{~S}$

$8 \mathrm{R}_{1}==\mathrm{O}, \mathrm{R}_{2}=\mathrm{H}, \mathrm{R}_{3}=\mathrm{H}, \mathrm{R}_{4}=\mathrm{H}, \Delta 1(2), \Delta 7(8), 24 \mathrm{~S}$

$13 \mathrm{R}_{1}=\mathrm{OH}, \mathrm{R}_{2}=\mathrm{H}, \mathrm{R}_{3}=\mathrm{H}, \mathrm{R}_{4}=\mathrm{H}, 24 \mathrm{~S}$

$14 \mathrm{R}_{1}=\mathrm{OH}, \mathrm{R}_{2}=\mathrm{H}, \mathrm{R}_{3}=\mathrm{H}, \mathrm{R}_{4}=\mathrm{H}, \Delta 7(8), 24 \mathrm{~S}$

$15 \mathrm{R}_{1}=\mathrm{OH}, \mathrm{R}_{2}=\mathrm{H}, \mathrm{R}_{3}=\mathrm{H}, \mathrm{R}_{4}=\mathrm{Ac}, \Delta 7(8), 24 \mathrm{~S}$

$16 \mathrm{R}_{1}==\mathrm{O}, \mathrm{R}_{2}=\mathrm{H}, \mathrm{R}_{3}=\mathrm{H}, \mathrm{R}_{4}=\mathrm{H}, 24 \mathrm{~S}$

$17 \mathrm{R}_{\mathrm{l}}==\mathrm{O}, \mathrm{R}_{2}=\mathrm{H}, \mathrm{R}_{3}=\mathrm{H}, \mathrm{R}_{4}=\mathrm{H}, \Delta 7(8), 24 R$

$18 \mathrm{R}_{1}==\mathrm{O}, \mathrm{R}_{2}=\mathrm{H}_{1} \mathrm{R}_{3}=\mathrm{H}_{1} \mathrm{R}_{4}=\mathrm{H}, \triangle \mathrm{I}(2), 24 \mathrm{~S}$

$19 \mathrm{R}_{1}=O-2^{\prime}-O$-acetyl- $\alpha$-L-arabinose, $\mathrm{R}_{3}=\mathrm{H}, \mathrm{R}_{3}=\mathrm{H}, \mathrm{R}_{4}=\mathrm{Ac}, 24 \mathrm{~S}$

$27 \mathrm{R}_{1}=O_{-} \alpha$-L-arabinose, $\mathrm{R}_{2}=\mathrm{H}, \mathrm{R}_{3}=\mathrm{H}, \mathrm{R}_{4}=\mathrm{H}, 24 \mathrm{~S}$

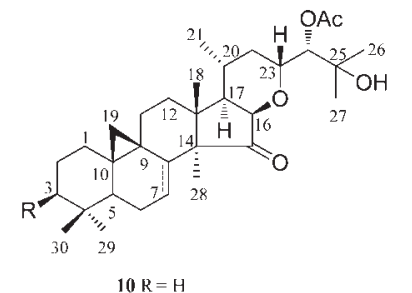

$11 \mathrm{R}=\mathrm{H}, \Delta 7(8)$

$24 \mathrm{R}=0-2^{\prime}-O$-acetyl- $\alpha$-L-arabinose

$29 \mathrm{R}=O$-acetyl $-\alpha-\mathrm{L}-$ arabinosc

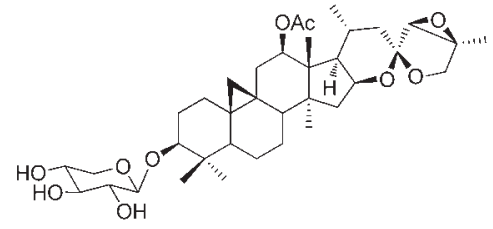

21

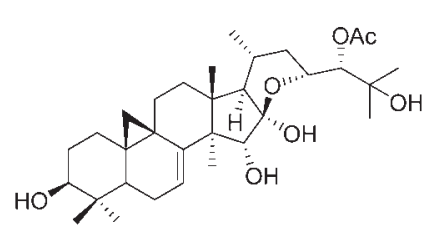

23

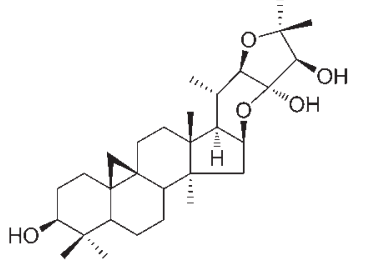

26

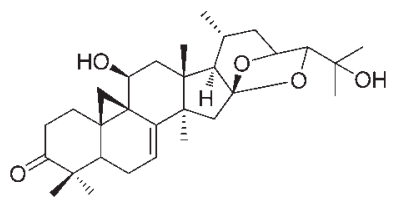

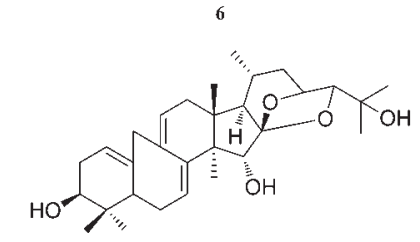

9

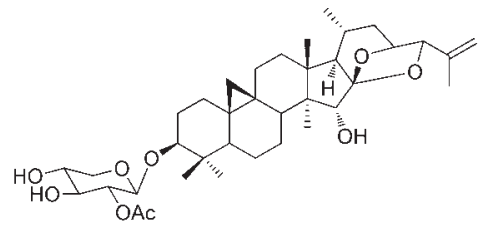

20

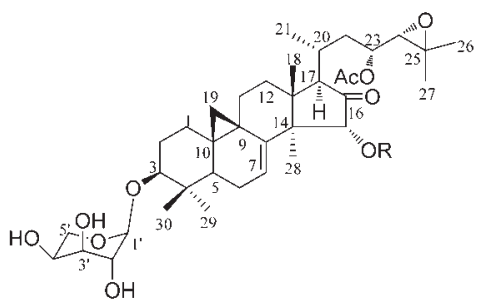

$12 \mathrm{R}=\mathrm{Ac}$

$30 \mathrm{R}=\mathrm{H}$

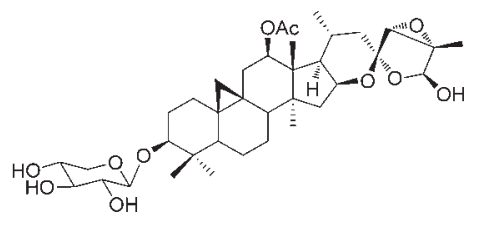

22

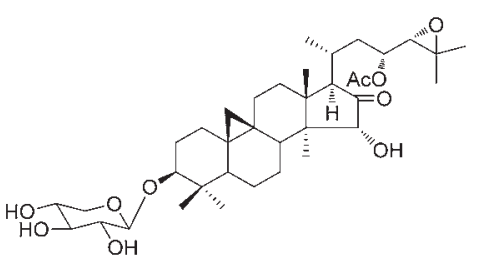

25

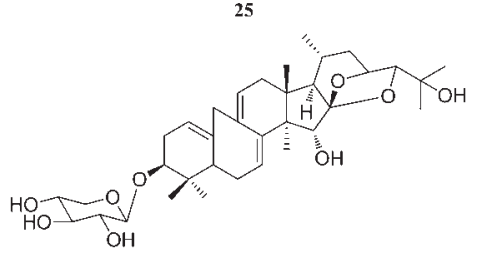

28

Fig. 1 Structures of compounds 1-30. 
Table $1{ }^{1} \mathrm{H}$ NMR data of compounds $1-12$ in pyridine- $d_{5}$ at $500 \mathrm{MHz}$.

\begin{tabular}{|c|c|c|c|c|c|c|c|c|c|c|c|c|}
\hline Proton & 1 & 2 & 3 & 4 & 5 & 6 & 7 & 8 & 9 & 10 & 11 & 12 \\
\hline 1 & $\begin{array}{l}1.53^{a} \\
1.17^{a}\end{array}$ & $\begin{array}{l}1.54 \mathrm{~m} \\
1.22^{a}\end{array}$ & $\begin{array}{l}1.54 \mathrm{~m} \\
1.16^{a}\end{array}$ & $\begin{array}{l}1.70 \mathrm{~m} \\
1.35 \mathrm{~m}\end{array}$ & $\begin{array}{l}2.78 \mathrm{~m} \\
1.71 \mathrm{~m}\end{array}$ & $\begin{array}{l}2.99 \mathrm{~m} \\
1.88 \mathrm{~m}\end{array}$ & $\begin{array}{l}1.85 \mathrm{~m} \\
1.54 \mathrm{~m}\end{array}$ & $\begin{array}{l}6.71 d \\
(10.0)\end{array}$ & $\begin{array}{l}5.54 \\
\text { brs }\end{array}$ & $\begin{array}{l}1.53 \mathrm{~m} \\
1.14 \mathrm{~m}\end{array}$ & $\begin{array}{l}1.68 \mathrm{~m} \\
1.24 \mathrm{~m}\end{array}$ & $\begin{array}{l}1.64 \mathrm{~m} \\
1.30^{a}\end{array}$ \\
\hline 2 & $\begin{array}{l}2.28 \mathrm{~m} \\
1.87 \mathrm{~m}\end{array}$ & $\begin{array}{l}2.34 \mathrm{~m} \\
1.96^{a}\end{array}$ & $\begin{array}{l}2.34 \text { dd } \\
(4.0 \\
16.0) \\
1.95^{a}\end{array}$ & $\begin{array}{l}1.92 \mathrm{~m} \\
1.86 \\
\text { brs }\end{array}$ & $\begin{array}{l}2.11 \mathrm{~m} \\
1.93 \mathrm{~m}\end{array}$ & $\begin{array}{l}2.84 \mathrm{~m} \\
2.35 \mathrm{~m}\end{array}$ & $\begin{array}{l}2.75 \\
\text { ddd } \\
(6.5) \\
2.29 \mathrm{~m}\end{array}$ & $\begin{array}{l}6.16 \mathrm{~d} \\
(10.0)\end{array}$ & $\begin{array}{l}2.52 \mathrm{~m} \\
2.26 \mathrm{~m}\end{array}$ & $\begin{array}{l}1.99 \mathrm{~m} \\
1.86 \mathrm{dd} \\
(3.2, \\
10.0)\end{array}$ & $\begin{array}{l}1.95 \mathrm{~m} \\
1.89 \mathrm{~m}\end{array}$ & $\begin{array}{l}2.36 \mathrm{~m} \\
1.95 \mathrm{~m}\end{array}$ \\
\hline 3 & $\begin{array}{l}3.39 \\
\text { dd } \\
(4.0, \\
11.5)\end{array}$ & $\begin{array}{l}3.50 \text { dd } \\
(5.0 \\
14.5)\end{array}$ & $\begin{array}{l}3.47 \mathrm{dd} \\
(5.0 \\
14.2)\end{array}$ & $\begin{array}{l}3.50 \mathrm{dd} \\
(3.5 \\
11.0)\end{array}$ & $\begin{array}{l}3.57 \mathrm{dd} \\
(5.0 \\
10.5)\end{array}$ & & & & $3.78 \mathrm{~m}$ & $3.48 \mathrm{~m}$ & $3.51 \mathrm{~m}$ & $\begin{array}{l}3.49 \text { dd } \\
(3.5 \\
10.5)\end{array}$ \\
\hline \multicolumn{13}{|l|}{4} \\
\hline 5 & $\begin{array}{l}1.28 \\
\text { dd } \\
(3.0, \\
12.3)\end{array}$ & $1.30^{a}$ & $1.29 \mathrm{~m}$ & $\begin{array}{l}1.25 \mathrm{dd} \\
(5.0 \\
12.0)\end{array}$ & $\begin{array}{l}1.31 \mathrm{dd} \\
(5.0, \\
12.0)\end{array}$ & $1.64 \mathrm{~m}$ & $1.54 \mathrm{~m}$ & $\begin{array}{l}1.84 \text { dd } \\
(5.0 \\
12.5)\end{array}$ & $2.36 \mathrm{~m}$ & $\begin{array}{l}1.25 \text { dd } \\
(3.2, \\
10.0)\end{array}$ & $1.20^{a}$ & $1.27^{a}$ \\
\hline 7 & $\begin{array}{l}2.06 \mathrm{~m} \\
1.11^{a}\end{array}$ & $\begin{array}{l}2.07 \mathrm{~m} \\
1.07^{a}\end{array}$ & $\begin{array}{l}2.09 \mathrm{~m} \\
1.04^{a}\end{array}$ & $\begin{array}{l}6.20 \mathrm{~d} \\
(7.0)\end{array}$ & $\begin{array}{l}6.21 \mathrm{~d} \\
(6.5)\end{array}$ & $5.23 \mathrm{~m}$ & $\begin{array}{l}6.05 \\
\text { brd } \\
(7.0)\end{array}$ & $\begin{array}{l}6.11 \mathrm{~d} \\
(7.0)\end{array}$ & $\begin{array}{l}5.52 \\
\text { brd } \\
(8.0)\end{array}$ & $\begin{array}{l}2.27 \mathrm{~m} \\
1.95 \mathrm{~m}\end{array}$ & $\begin{array}{l}6.36 \\
\text { brd } \\
(6.5)\end{array}$ & $\begin{array}{l}5.33 \mathrm{~d} \\
(7.0)\end{array}$ \\
\hline 8 & $1.65^{a}$ & $1.66^{a}$ & $1.66^{a}$ & & & & & & & $\begin{array}{l}1.72 \\
\text { brd } \\
(10.4)\end{array}$ & & \\
\hline \multicolumn{13}{|l|}{9} \\
\hline 11 & $\begin{array}{l}2.06 \mathrm{~m} \\
1.01 \mathrm{~m}\end{array}$ & $\begin{array}{l}2.07 \mathrm{~m} \\
1.15^{a}\end{array}$ & $\begin{array}{l}2.09 \mathrm{~m} \\
1.15^{a}\end{array}$ & $\begin{array}{l}2.96 \mathrm{dd} \\
(9.0 \\
15.0) \\
1.54^{a}\end{array}$ & $\begin{array}{l}4.58 \\
\text { brd } \\
(8.5)\end{array}$ & $4.53 \mathrm{~m}$ & $\begin{array}{l}2.13 \mathrm{~m} \\
1.16^{a}\end{array}$ & $\begin{array}{l}2.23 \mathrm{~m} \\
1.40 \mathrm{~m}\end{array}$ & $\begin{array}{l}5.39 \\
\text { brd } \\
(5.0)\end{array}$ & $\begin{array}{l}2.23 \mathrm{~m} \\
1.05^{a}\end{array}$ & $\begin{array}{l}1.98 \mathrm{~m} \\
1.13^{a}\end{array}$ & $\begin{array}{l}2.11 \mathrm{~m} \\
1.16 \mathrm{~m}\end{array}$ \\
\hline 12 & $\begin{array}{l}1.65^{a} \\
1.53^{a}\end{array}$ & $\begin{array}{l}1.66^{a} \\
1.52 \mathrm{~m}\end{array}$ & $\begin{array}{l}1.64^{a} \\
1.52 \mathrm{~m}\end{array}$ & $\begin{array}{l}4.36 \mathrm{t} \\
(6.5)\end{array}$ & $\begin{array}{l}2.74 \mathrm{~m} \\
2.11 \mathrm{~m}\end{array}$ & $\begin{array}{l}2.73 \mathrm{~m} \\
1.99 \mathrm{~m}\end{array}$ & $\begin{array}{l}1.81 \mathrm{~m} \\
1.68 \mathrm{~m}\end{array}$ & $\begin{array}{l}1.81 \mathrm{~m} \\
1.70 \mathrm{~m}\end{array}$ & $\begin{array}{l}2.21 \mathrm{~m} \\
1.94 \mathrm{dd} \\
(6.0, \\
12.5)\end{array}$ & $\begin{array}{l}1.58 \mathrm{~m} \\
1.37 \mathrm{~m}\end{array}$ & $\begin{array}{l}1.65 \mathrm{~m} \\
1.29 \mathrm{~m}\end{array}$ & $\begin{array}{l}1.89 \mathrm{~m} \\
1.78 \mathrm{~m}\end{array}$ \\
\hline \multicolumn{13}{|l|}{13} \\
\hline 15 & $\begin{array}{l}4.26 \\
\text { brs }\end{array}$ & $\begin{array}{l}4.27 \\
\text { brs }\end{array}$ & $\begin{array}{l}4.25 \\
\text { brs }\end{array}$ & $\begin{array}{l}4.76 \\
\text { brs }\end{array}$ & $4.62 \mathrm{~s}$ & $\begin{array}{l}2.46 \mathrm{~d} \\
(13.0) \\
2.28 \mathrm{~d} \\
(13.0)\end{array}$ & $\begin{array}{l}4.55 \\
\text { brd } \\
(8.0)\end{array}$ & $\begin{array}{l}4.54 d \\
(9.0)\end{array}$ & $\begin{array}{l}2.36 \mathrm{~m} \\
2.09 \mathrm{~m}\end{array}$ & & & $5.92 \mathrm{~s}$ \\
\hline 16 & & & & & & & & & & $\begin{array}{l}3.79 d \\
(9.6)\end{array}$ & $\begin{array}{l}3.83 \mathrm{~d} \\
(11.2)\end{array}$ & \\
\hline 17 & $1.48^{a}$ & $\begin{array}{l}1.44 \\
\text { brd } \\
(14.0)\end{array}$ & $\begin{array}{l}1.45 \\
\text { brd } \\
(14.0)\end{array}$ & $\begin{array}{l}1.86 \\
\text { brs }\end{array}$ & $1.48^{a}$ & $\begin{array}{l}1.59 \mathrm{~d} \\
(12.0)\end{array}$ & $1.50^{a}$ & $1.49^{a}$ & $1.53^{a}$ & $1.52 \mathrm{~m}$ & $1.20 \mathrm{~m}$ & $\begin{array}{l}2.34 \mathrm{~d} \\
(8.0)\end{array}$ \\
\hline 18 & $1.13 \mathrm{~s}$ & $1.13 \mathrm{~s}$ & $1.13 \mathrm{~s}$ & $1.51 \mathrm{~s}$ & $1.27 \mathrm{~s}$ & $1.26 \mathrm{~s}$ & $1.16 \mathrm{~s}$ & $1.10 \mathrm{~s}$ & $\begin{array}{l}0.81 \\
\text { brs }\end{array}$ & $1.18 \mathrm{~s}$ & $1.20 \mathrm{~s}$ & $1.29 \mathrm{~s}$ \\
\hline 19 & $\begin{array}{l}0.45 \\
\text { brs } \\
0.21 \\
\text { brs }\end{array}$ & $\begin{array}{l}0.50 \mathrm{~d} \\
(4.0) \\
0.25 \mathrm{~d} \\
(4.0)\end{array}$ & $\begin{array}{l}0.52 \mathrm{~d} \\
(4.0) \\
0.28 \mathrm{~d} \\
(4.0)\end{array}$ & $\begin{array}{l}1.19^{a} \\
0.73 d \\
(4.0)\end{array}$ & $\begin{array}{l}1.97 \mathrm{~d} \\
(4.0) \\
1.07 \\
\text { brd } \\
(2.5)\end{array}$ & $\begin{array}{l}2.11 \mathrm{~d} \\
(4.0) \\
1.11 \mathrm{~d} \\
(4.0)\end{array}$ & $\begin{array}{l}1.19^{a} \\
0.67 \mathrm{~d} \\
(4.0)\end{array}$ & $\begin{array}{l}1.52 \mathrm{~d} \\
(4.8) \\
1.04 \mathrm{~d} \\
(4.0)\end{array}$ & $\begin{array}{l}3.24 \mathrm{~d} \\
(13.5) \\
3.15 \mathrm{~d} \\
(13.5)\end{array}$ & $\begin{array}{l}0.52 \mathrm{~d} \\
(3.6) \\
0.29 \mathrm{~d} \\
(3.6)\end{array}$ & $\begin{array}{l}1.07^{a} \\
0.48 \mathrm{~d} \\
(4.0)\end{array}$ & $\begin{array}{l}0.99^{a} \\
0.51 \mathrm{~d} \\
(3.5)\end{array}$ \\
\hline 20 & $1.65^{a}$ & $1.67^{a}$ & $1.59 \mathrm{~m}$ & $1.86 \mathrm{~m}$ & $\begin{array}{l}1.64 \\
\text { brd } \\
(10.5)\end{array}$ & $\begin{array}{l}1.66 \\
\text { brd } \\
(9.5)\end{array}$ & $1.70 \mathrm{~m}$ & $1.68 \mathrm{~m}$ & $1.60 \mathrm{~m}$ & $1.80 \mathrm{~m}$ & $1.81 \mathrm{~m}$ & $2.10 \mathrm{~m}$ \\
\hline 21 & $\begin{array}{l}0.84 \mathrm{~d} \\
(7.0)\end{array}$ & $\begin{array}{l}0.83 d \\
(6.4)\end{array}$ & $\begin{array}{l}0.84 d \\
(6.4)\end{array}$ & $\begin{array}{l}1.41 \mathrm{~d} \\
(5.5)\end{array}$ & $\begin{array}{l}0.82 \mathrm{~d} \\
(6.0)\end{array}$ & $\begin{array}{l}0.83 \mathrm{~d} \\
(6.0)\end{array}$ & $\begin{array}{l}0.89 \mathrm{~d} \\
(6.5)\end{array}$ & $\begin{array}{l}0.89 \mathrm{~d} \\
(7.0)\end{array}$ & $\begin{array}{l}0.81 \\
\text { brs }\end{array}$ & $\begin{array}{l}0.91 \mathrm{~d} \\
(7.2)\end{array}$ & $\begin{array}{l}0.93 d \\
(6.5)\end{array}$ & $\begin{array}{l}1.19 d \\
(6.5)\end{array}$ \\
\hline 22 & $\begin{array}{l}2.26 \mathrm{~m} \\
1.01 \mathrm{~m}\end{array}$ & $\begin{array}{l}2.25 \mathrm{~m} \\
0.97 \mathrm{~m}\end{array}$ & $\begin{array}{l}2.26 \mathrm{~m} \\
0.95 \mathrm{~m}\end{array}$ & $\begin{array}{l}2.42 \mathrm{~m} \\
1.16^{a}\end{array}$ & $\begin{array}{l}2.22 \mathrm{~m} \\
0.99 \mathrm{~m}\end{array}$ & $\begin{array}{l}2.24 \mathrm{~m} \\
0.99 \mathrm{t} \\
(11.5)\end{array}$ & $\begin{array}{l}2.30 \mathrm{~m} \\
1.07 \mathrm{~m}\end{array}$ & $\begin{array}{l}2.28 \mathrm{~m} \\
1.07 \mathrm{~m}\end{array}$ & $\begin{array}{l}2.21 \mathrm{~m} \\
0.96^{a}\end{array}$ & $\begin{array}{l}1.72 \\
\text { brd } \\
(10.4) \\
1.44 \mathrm{~m}\end{array}$ & $\begin{array}{l}1.73 \mathrm{~m} \\
1.47 \mathrm{~m}\end{array}$ & $\begin{array}{l}2.77 \mathrm{t} \\
(12.0) \\
1.68 \mathrm{~m}\end{array}$ \\
\hline 23 & $\begin{array}{l}4.74 d \\
(9.0)\end{array}$ & $\begin{array}{l}4.59 \mathrm{~d} \\
(11.5)\end{array}$ & $\begin{array}{l}4.59 \\
\text { brd } \\
(12.0)\end{array}$ & $\begin{array}{l}4.80 \mathrm{~d} \\
(9.0)\end{array}$ & $\begin{array}{l}4.73 d \\
(9.0)\end{array}$ & $\begin{array}{l}4.79 d \\
(8.5)\end{array}$ & $\begin{array}{l}4.78 \\
\text { brd } \\
(9.0)\end{array}$ & $\begin{array}{l}4.77 t \\
(8.0)\end{array}$ & $\begin{array}{l}4.77 \\
\text { brd } \\
(9.0)\end{array}$ & $\begin{array}{l}4.25 \\
\text { brd } \\
(11.2)\end{array}$ & $\begin{array}{l}4.27 d \\
(11.5)\end{array}$ & $\begin{array}{l}5.38 \\
\text { brt } \\
(9.5)\end{array}$ \\
\hline
\end{tabular}




\begin{tabular}{|c|c|c|c|c|c|c|c|c|c|c|c|c|}
\hline Proton & 1 & 2 & 3 & 4 & 5 & 6 & 7 & 8 & 9 & 10 & 11 & 12 \\
\hline 26 & $1.48 \mathrm{~s}$ & $1.68 \mathrm{~s}$ & $1.68 \mathrm{~s}$ & $1.52 \mathrm{~s}$ & $1.47 \mathrm{~s}$ & $1.51 \mathrm{~s}$ & $1.48 \mathrm{~s}$ & $1.49 \mathrm{~s}$ & $1.52 \mathrm{~s}$ & $1.61 \mathrm{~s}$ & $1.62 \mathrm{~s}$ & $1.25 \mathrm{~s}$ \\
\hline 27 & $1.46 \mathrm{~s}$ & $1.66 \mathrm{~s}$ & $1.66 \mathrm{~s}$ & $1.51 \mathrm{~s}$ & $1.45 \mathrm{~s}$ & $1.46 \mathrm{~s}$ & $1.49 \mathrm{~s}$ & $1.48 \mathrm{~s}$ & $1.47 \mathrm{~s}$ & $1.61 \mathrm{~s}$ & $1.63 \mathrm{~s}$ & $1.39 \mathrm{~s}$ \\
\hline 28 & $1.16 \mathrm{~s}$ & $1.18 \mathrm{~s}$ & $1.18 \mathrm{~s}$ & $1.46 \mathrm{~s}$ & $1.36 \mathrm{~s}$ & $1.39 \mathrm{~s}$ & $1.38 \mathrm{~s}$ & $1.36 \mathrm{~s}$ & $1.28 \mathrm{~s}$ & $1.01 \mathrm{~s}$ & $1.13 \mathrm{~s}$ & $1.34 \mathrm{~s}$ \\
\hline 29 & $1.08 \mathrm{~s}$ & $1.31 \mathrm{~s}$ & $1.22 \mathrm{~s}$ & $1.17 \mathrm{~s}$ & $1.19 \mathrm{~s}$ & $1.16 \mathrm{~s}$ & $1.04 \mathrm{~s}$ & $1.15 \mathrm{~s}$ & $1.31 \mathrm{~s}$ & $1.19 \mathrm{~s}$ & $1.21 \mathrm{~s}$ & $1.31 \mathrm{~s}$ \\
\hline 30 & $0.95 \mathrm{~s}$ & $1.04 \mathrm{~s}$ & $1.00 \mathrm{~s}$ & $1.07 \mathrm{~s}$ & $1.14 \mathrm{~s}$ & $1.12 \mathrm{~s}$ & $1.07 \mathrm{~s}$ & $0.99 \mathrm{~s}$ & $0.98 \mathrm{~s}$ & $1.05 \mathrm{~s}$ & $1.07 \mathrm{~s}$ & $1.02 \mathrm{~s}$ \\
\hline \multicolumn{13}{|l|}{ 3-Ara } \\
\hline $1^{\prime}$ & $\begin{array}{l}4.79 \mathrm{~d} \\
(7.5)\end{array}$ & $\begin{array}{l}4.79 d \\
(9.0)\end{array}$ & $\begin{array}{l}4.88 \mathrm{~d} \\
(8.0)\end{array}$ & & & & & & & & & $\begin{array}{l}4.79 \mathrm{~d} \\
(7.0)\end{array}$ \\
\hline $2^{\prime}$ & $\begin{array}{l}5.99 \mathrm{t} \\
(8.5)\end{array}$ & $\begin{array}{l}4.38 \mathrm{t} \\
(10.0)\end{array}$ & $\begin{array}{l}4.63 \\
\text { brd } \\
(6.0)\end{array}$ & & & & & & & & & $4.18 \mathrm{~m}$ \\
\hline $3^{\prime}$ & $\begin{array}{l}4.23 \\
\text { brd } \\
(9.5)\end{array}$ & $4.24 m$ & $\begin{array}{l}5.48 \mathrm{dd} \\
(4.5, \\
11.0)\end{array}$ & & & & & & & & & $\begin{array}{l}4.47 \mathrm{t} \\
(8.0)\end{array}$ \\
\hline $4^{\prime}$ & $\begin{array}{l}4.30 \\
\text { brs }\end{array}$ & $\begin{array}{l}5.50 \\
\text { brs }\end{array}$ & $\begin{array}{l}4.57 \\
\text { brs }\end{array}$ & & & & & & & & & $\begin{array}{l}4.33 \\
\text { brs }\end{array}$ \\
\hline $5^{\prime}$ & $\begin{array}{l}4.28 \mathrm{~m} \\
3.79 \\
\text { brd } \\
(12.0)\end{array}$ & $\begin{array}{l}4.24 \mathrm{~m} \\
3.81 \mathrm{~d} \\
(16.0)\end{array}$ & $\begin{array}{l}4.30 \mathrm{~m} \\
3.85 \mathrm{~d} \\
(13.5)\end{array}$ & & & & & & & & & $\begin{array}{l}4.31 \\
\text { brd } \\
(9.5) \\
3.79 \mathrm{~d} \\
(12.5)\end{array}$ \\
\hline $15-\mathrm{OCOCH}_{3}$ & & & & & & & & & & & & $2.26 \mathrm{~s}$ \\
\hline $23-\mathrm{OCOCH}_{3}$ & & & & & & & & & & & & $2.02 \mathrm{~s}$ \\
\hline $24-\mathrm{OCOCH}_{3}$ & & & & & & & & & & $2.16 \mathrm{~s}$ & $2.18 \mathrm{~s}$ & \\
\hline $25-\mathrm{OCOCH}_{3}$ & & $1.96 \mathrm{~s}$ & $1.95 \mathrm{~s}$ & & & & & & & & & \\
\hline $\mathbf{3}^{\prime}-\mathrm{OCOCH}_{3}$ & & & $1.96 \mathrm{~s}$ & & & & & & & & & \\
\hline $4^{\prime}-\mathrm{OCOCH}_{3}$ & & $1.96 \mathrm{~s}$ & & & & & & & & & & \\
\hline $\begin{array}{l}4^{\prime}- \\
\mathrm{OCOCH}=\mathrm{C}- \\
\mathrm{H}-\mathrm{CH}_{3}\end{array}$ & $\begin{array}{l}6.05 d \\
(15.5)\end{array}$ & & & & & & & & & & & \\
\hline $\begin{array}{l}4^{\prime}- \\
\mathrm{OCOCH}=\mathrm{C}- \\
\underline{\mathrm{H}}-\mathrm{CH}_{3}\end{array}$ & $7.09 \mathrm{~m}$ & & & & & & & & & & & \\
\hline $\begin{array}{l}4^{\prime}- \\
\mathrm{OCOCH}=\mathrm{C}- \\
\mathrm{H}-\mathrm{CH}_{2}\end{array}$ & $\begin{array}{l}1.63 \mathrm{~d} \\
(6.5)\end{array}$ & & & & & & & & & & & \\
\hline
\end{tabular}

Chemical shifts are in $\delta$ scale with / values in parentheses. ${ }^{a}$ Signals overlapped

9,10-Seco-1(10),7(8),9(11)-trien-cimigenol (9): A white powder; $[\alpha]_{\mathrm{D}}^{24}=5.73($ c $0.05, \mathrm{MeOH}) ; \mathrm{IR}(\mathrm{KBr}): v_{\max }=3432,2947,2852$, $1624,1460,1383,1070,978 \mathrm{~cm}^{-1} ;{ }^{1} \mathrm{H}\left(\mathrm{C}_{5} \mathrm{D}_{5} \mathrm{~N}, 500 \mathrm{MHz}\right)$ and ${ }^{13} \mathrm{C}$ $\operatorname{NMR}\left(\mathrm{C}_{5} \mathrm{D}_{5} \mathrm{~N}, 150 \mathrm{MHz}\right)$ spectra, see $\odot$ Tables 1 and 2; ESIMS: $m /$ $z=469[\mathrm{M}+\mathrm{H}]^{+}$; HR-TOF-ESIMS: $m / z=469.3311$ (calc. for $\left.\mathrm{C}_{30} \mathrm{H}_{45} \mathrm{O}_{4}, 469.3317\right)$.

24-O-Acetylisodahurinol (10): A white powder; $[\alpha]_{\mathrm{D}}^{24}=2.09(c$ 0.07, MeOH); IR (KBr): $v_{\max }=3473,2946,2862,1738,1471$, 1377, 1024, $994 \mathrm{~cm}^{-1} ;{ }^{1} \mathrm{H}\left(\mathrm{C}_{5} \mathrm{D}_{5} \mathrm{~N}, 500 \mathrm{MHz}\right)$ and ${ }^{13} \mathrm{C} \mathrm{NMR}$ $\left(\mathrm{C}_{5} \mathrm{D}_{5} \mathrm{~N}, 125 \mathrm{MHz}\right)$ spectra, see Tables 1 and 2; ESIMS: $\mathrm{m} /$ $z=553[\mathrm{M}+\mathrm{Na}]^{+}$; HR-TOF-ESIMS: $m / z=553.3494$ (calc. for $\left.\mathrm{C}_{32} \mathrm{H}_{50} \mathrm{O}_{6} \mathrm{Na}, 553.3505\right)$.

24-O-Acetyl-7(8)-en-isodahurinol (11): A white powder; $[\alpha]_{\mathrm{D}}^{24}=$ -4.80 (c 0.05, MeOH); IR (KBr): $v_{\max }=3437,2920,2875,1736$, $1640,1472,1375,1026,996 \mathrm{~cm}^{-1} ;{ }^{1} \mathrm{H}\left(\mathrm{C}_{5} \mathrm{D}_{5} \mathrm{~N}, 500 \mathrm{MHz}\right)$ and ${ }^{13} \mathrm{C}$ $\operatorname{NMR}\left(\mathrm{C}_{5} \mathrm{D}_{5} \mathrm{~N}, 125 \mathrm{MHz}\right)$ spectra, see 0 Tables 1 and 2; ESIMS: $m /$ $z=551[\mathrm{M}+\mathrm{Na}]^{+}$; HR-TOF-ESIMS: $m / z=551.3346$ (calc. for $\mathrm{C}_{32} \mathrm{H}_{48} \mathrm{O}_{6} \mathrm{Na}, 551.3348$ ).

23-0-Diacetyl-7(8)-en-shengmanol-3-O- $\alpha$-L-arabinopyranoside

(12): A white powder; $[\alpha]_{\mathrm{D}}^{24}=-7.69(\mathrm{MeOH}$, c 0.07); IR (KBr): $v_{\max }=3473,2942,2871,1736,1643,1458,1376,1024,972 \mathrm{~cm}^{-1}$; ${ }^{1} \mathrm{H}\left(\mathrm{C}_{5} \mathrm{D}_{5} \mathrm{~N}, 500 \mathrm{MHz}\right)$ and ${ }^{13} \mathrm{C} \mathrm{NMR}\left(\mathrm{C}_{5} \mathrm{D}_{5} \mathrm{~N}, 150 \mathrm{MHz}\right)$ spectra, see $\bigcirc$ Tables 1 and 2; ESIMS: $m / z=725[\mathrm{M}+\mathrm{Na}]^{+}$; HR-TOF-ESIMS: $m / z=725.3895[\mathrm{M}+\mathrm{Na}]^{+}$(calc. for $\mathrm{C}_{39} \mathrm{H}_{58} \mathrm{O}_{11} \mathrm{Na}, 725.3876$ ).

\section{Hydrolysis and identification of the sugar moieties in} compounds $1,2,3$, and 12

Compound 1 ( $4.0 \mathrm{mg}$ ) together with 2 and 3 (3.0 $\mathrm{mg}$ of each) were individually dissolved in $\mathrm{MeOH}(5 \mathrm{~mL})$, then $4 \% \mathrm{~K}_{2} \mathrm{CO}_{3}(5 \mathrm{~mL})$ was added, and each solution was stirred at rt overnight. Each solution was neutralized by $10 \%$ HOAc and extracted with EtOAc $(3 \times 15 \mathrm{~mL})$. Each EtOAc extract, after removal of solvent, was dissolved in $\mathrm{MeOH}(5 \mathrm{~mL})$ and refluxed with $0.5 \mathrm{~N} \mathrm{HCl}(3 \mathrm{~mL}$ ) for $4 \mathrm{~h}$ [7]. Compound 12 (4.0 mg), by contrast, was directly dissolved in $\mathrm{MeOH}(5 \mathrm{~mL})$ and refluxed with $0.5 \mathrm{~N} \mathrm{HCl}$ (3 mL) for $4 \mathrm{~h}$. Each reaction mixture was diluted with $\mathrm{H}_{2} \mathrm{O}$ and extracted with $\mathrm{CHCl}_{3}$ $(3 \times 10 \mathrm{~mL})$. Each aqueous layer was then neutralized by $\mathrm{Ag}_{2} \mathrm{CO}_{3}$, and the formed precipitation was filtered to give a monosaccharide, which had an $\mathrm{Rf}$ (EtOAc- $\mathrm{CHCl}_{3}-\mathrm{MeOH}-\mathrm{H}_{2} \mathrm{O}, 3: 2: 2: 1$ ) and specific rotation $[\alpha]_{\mathrm{D}}^{20}+82.78(c 0.05, \mathrm{MeOH})$ corresponding to those of L-arabinose (Sigma-Aldrich). 
Table $2{ }^{13} \mathrm{C}$ NMR data of compounds $\mathbf{1 - 1 2}$ in pyridine- $d_{5}$ at $125 \mathrm{MHz}(\mathbf{2}-\mathbf{5}, \mathbf{7}, \mathbf{8}, \mathbf{1 0}, \mathbf{1 1})$ and $150 \mathrm{MHz}(\mathbf{1}, \mathbf{6}, \mathbf{9}, \mathbf{1 2})$.

\begin{tabular}{|c|c|c|c|c|c|c|c|c|c|c|c|c|}
\hline C & 1 & 2 & 3 & 4 & 5 & 6 & 7 & 8 & 9 & 10 & 11 & 12 \\
\hline 1 & $32.8 \mathrm{t}$ & $32.4 \mathrm{t}$ & $32.4 \mathrm{t}$ & $30.8 \mathrm{t}$ & $27.7 t$ & $29.5 t$ & $31.7 t$ & $152.6 \mathrm{~d}$ & $121.0 \mathrm{~d}$ & $32.7 t$ & $31.3 t$ & $30.6 \mathrm{t}$ \\
\hline 2 & $30.4 \mathrm{t}$ & $30.0 \mathrm{t}$ & $29.9 t$ & $30.7 t$ & $31.1 \mathrm{t}$ & $36.9 \mathrm{t}$ & $36.9 t$ & $127.2 \mathrm{~d}$ & $33.7 t$ & $31.3 \mathrm{t}$ & $30.7 t$ & $29.9 t$ \\
\hline 3 & $89.2 \mathrm{~d}$ & $88.9 \mathrm{~d}$ & $88.7 \mathrm{~d}$ & $77.8 \mathrm{~d}$ & $78.1 \mathrm{~d}$ & $216.1 \mathrm{~s}$ & $214.9 s$ & $203.8 \mathrm{~s}$ & $74.2 \mathrm{~d}$ & $77.9 \mathrm{~d}$ & $77.6 \mathrm{~d}$ & $88.5 \mathrm{~d}$ \\
\hline 4 & $41.6 \mathrm{~s}$ & $41.3 \mathrm{~s}$ & $41.3 \mathrm{~s}$ & $40.2 \mathrm{~s}$ & $40.5 \mathrm{~s}$ & $49.5 \mathrm{~s}$ & $48.9 \mathrm{~s}$ & $45.1 \mathrm{~s}$ & $39.6 \mathrm{~s}$ & $41.1 \mathrm{~s}$ & $40.2 \mathrm{~s}$ & $40.9 \mathrm{~s}$ \\
\hline 5 & $47.9 \mathrm{~d}$ & $47.6 \mathrm{~d}$ & $47.6 \mathrm{~d}$ & $42.4 \mathrm{~d}$ & $43.6 \mathrm{~d}$ & $45.9 \mathrm{~d}$ & $44.2 \mathrm{~d}$ & $40.4 d$ & $51.7 \mathrm{~d}$ & $47.3 \mathrm{~d}$ & $41.9 \mathrm{~d}$ & $42.8 \mathrm{~d}$ \\
\hline 6 & $21.5 t$ & $21.1 \mathrm{t}$ & $21.1 \mathrm{t}$ & $22.0 \mathrm{t}$ & $22.2 \mathrm{t}$ & $22.7 t$ & $21.9 t$ & $21.8 \mathrm{t}$ & $38.5 t$ & $21.0 \mathrm{t}$ & $21.9 t$ & $22.2 \mathrm{t}$ \\
\hline 7 & $26.9 t$ & $26.3 \mathrm{t}$ & $26.4 \mathrm{t}$ & $114.5 d$ & $115.2 d$ & $114.5 \mathrm{~d}$ & $114.2 \mathrm{~d}$ & $115.5 d$ & $125.1 \mathrm{~d}$ & $26.2 \mathrm{t}$ & $115.0 \mathrm{~d}$ & $115.6 \mathrm{~d}$ \\
\hline 8 & $49.1 \mathrm{~d}$ & $48.6 \mathrm{~d}$ & $48.6 \mathrm{~d}$ & $147.4 \mathrm{~s}$ & $147.3 \mathrm{~s}$ & $149.1 \mathrm{~s}$ & $147.9 \mathrm{~s}$ & 147.4 & $144.8 \mathrm{~s}$ & $43.7 \mathrm{~d}$ & 141.9 & $146.6 \mathrm{~s}$ \\
\hline 9 & $20.5 \mathrm{~s}$ & $20.0 \mathrm{~s}$ & $20.1 \mathrm{~s}$ & $21.9 \mathrm{~s}$ & $28.0 \mathrm{~s}$ & $28.9 \mathrm{~s}$ & $22.1 \mathrm{~s}$ & $26.3 \mathrm{~s}$ & $138.3 \mathrm{~s}$ & $20.0 \mathrm{~s}$ & $21.0 \mathrm{~s}$ & $21.8 s$ \\
\hline 10 & $27.0 \mathrm{~s}$ & $26.5 \mathrm{~s}$ & $26.7 \mathrm{~s}$ & $28.3 \mathrm{~s}$ & $29.4 \mathrm{~s}$ & $29.4 \mathrm{~s}$ & $28.2 \mathrm{~s}$ & $32.8 \mathrm{~s}$ & $139.8 \mathrm{~s}$ & $27.4 \mathrm{~s}$ & $29.3 \mathrm{~s}$ & $29.1 \mathrm{~s}$ \\
\hline 11 & $26.8 \mathrm{t}$ & $26.4 \mathrm{t}$ & $26.5 \mathrm{t}$ & $40.3 t$ & $63.5 d$ & $63.7 d$ & $25.6 \mathrm{t}$ & $26.5 t$ & $122.1 \mathrm{~d}$ & $26.0 \mathrm{t}$ & $25.3 t$ & $25.3 t$ \\
\hline 12 & $34.5 \mathrm{t}$ & $34.0 \mathrm{t}$ & $34.1 \mathrm{t}$ & $72.5 d$ & $49.4 \mathrm{t}$ & $48.7 t$ & $33.9 \mathrm{t}$ & $33.8 \mathrm{t}$ & $38.6 \mathrm{t}$ & $31.4 \mathrm{t}$ & $30.6 \mathrm{t}$ & $33.4 \mathrm{t}$ \\
\hline 13 & $42.3 \mathrm{~s}$ & $41.8 \mathrm{~s}$ & $41.9 \mathrm{~s}$ & $47.1 \mathrm{~s}$ & $41.6 \mathrm{~s}$ & $46.0 \mathrm{~s}$ & $41.2 \mathrm{~s}$ & $41.3 \mathrm{~s}$ & $45.2 \mathrm{~s}$ & $40.0 \mathrm{~s}$ & $40.2 \mathrm{~s}$ & $41.9 \mathrm{~s}$ \\
\hline 14 & $47.7 \mathrm{~s}$ & $47.2 \mathrm{~s}$ & $47.3 \mathrm{~s}$ & $51.3 \mathrm{~s}$ & $50.7 \mathrm{~s}$ & $48.6 \mathrm{~s}$ & $50.6 \mathrm{~s}$ & $50.9 \mathrm{~s}$ & $48.3 \mathrm{~s}$ & $55.1 \mathrm{~s}$ & $56.4 \mathrm{~s}$ & $48.9 \mathrm{~s}$ \\
\hline 15 & $80.7 \mathrm{~d}$ & $80.2 d$ & $80.2 d$ & $78.1 \mathrm{~d}$ & $78.2 \mathrm{~d}$ & $45.8 \mathrm{t}$ & $78.1 \mathrm{~d}$ & $77.9 \mathrm{~d}$ & $44.3 t$ & $213.9 \mathrm{~s}$ & $211.2 \mathrm{~s}$ & $82.1 \mathrm{~d}$ \\
\hline 16 & $112.5 \mathrm{~s}$ & $112.4 \mathrm{~s}$ & $112.5 \mathrm{~s}$ & $112.5 \mathrm{~s}$ & $112.4 \mathrm{~s}$ & $114.9 \mathrm{~s}$ & $112.2 \mathrm{~s}$ & $112.1 \mathrm{~s}$ & $114.5 \mathrm{~s}$ & $84.3 \mathrm{~d}$ & $84.3 \mathrm{~d}$ & $214.2 \mathrm{~s}$ \\
\hline 17 & $60.0 \mathrm{~d}$ & $59.4 d$ & $59.4 d$ & $59.8 \mathrm{~d}$ & $58.9 \mathrm{~d}$ & $61.6 \mathrm{~d}$ & $59.4 \mathrm{~d}$ & $59.4 \mathrm{~s}$ & $60.4 d$ & $52.4 \mathrm{~d}$ & $52.3 d$ & $60.3 d$ \\
\hline 18 & $20.0 q$ & $19.5 q$ & $19.5 q$ & $13.2 \mathrm{q}$ & $21.0 \mathrm{q}$ & $21.4 \mathrm{q}$ & $21.7 q$ & $21.5 q$ & $17.8 \mathrm{q}$ & $20.3 q$ & $25.4 \mathrm{q}$ & $22.3 q$ \\
\hline 19 & $31.2 \mathrm{t}$ & $30.9 t$ & $30.9 t$ & $28.8 \mathrm{t}$ & $18.7 t$ & $18.7 t$ & $27.7 t$ & $30.3 t$ & $44.3 \mathrm{t}$ & $31.3 \mathrm{t}$ & $28.5 t$ & $30.4 t$ \\
\hline 20 & $24.5 d$ & $23.9 \mathrm{~d}$ & $23.9 \mathrm{~d}$ & $23.8 \mathrm{~d}$ & $24.0 \mathrm{~d}$ & $24.3 d$ & $24.0 \mathrm{~d}$ & $24.0 \mathrm{~d}$ & $24.4 \mathrm{~d}$ & $33.3 \mathrm{~d}$ & $33.0 \mathrm{~d}$ & $28.9 \mathrm{~d}$ \\
\hline 21 & $20.1 \mathrm{q}$ & $19.5 q$ & $19.5 q$ & $21.3 q$ & $19.6 q$ & $20.2 q$ & $19.7 \mathrm{q}$ & $19.7 q$ & $20.5 q$ & $20.0 q$ & $20.0 q$ & $20.3 q$ \\
\hline 22 & $38.6 \mathrm{t}$ & $37.9 \mathrm{t}$ & $37.9 \mathrm{t}$ & $38.5 t$ & $38.0 \mathrm{t}$ & $38.5 \mathrm{t}$ & $38.0 \mathrm{t}$ & $38.6 \mathrm{t}$ & $25.9 t$ & $38.8 \mathrm{t}$ & $38.7 t$ & $37.5 \mathrm{t}$ \\
\hline 23 & $72.3 d$ & $71.7 \mathrm{~d}$ & $71.7 \mathrm{~d}$ & $72.0 \mathrm{~d}$ & $72.0 \mathrm{~d}$ & $72.4 d$ & $72.1 \mathrm{~d}$ & $72.1 \mathrm{~d}$ & $72.3 d$ & $79.1 \mathrm{~d}$ & $79.0 \mathrm{~d}$ & $72.3 \mathrm{~d}$ \\
\hline 24 & $90.7 \mathrm{~d}$ & $86.8 \mathrm{~d}$ & $86.8 \mathrm{~d}$ & $90.1 \mathrm{~d}$ & $90.3 d$ & $91.1 \mathrm{~d}$ & $90.3 d$ & $90.3 d$ & $91.0 \mathrm{~d}$ & $79.8 \mathrm{~d}$ & $79.8 d$ & $65.6 \mathrm{~d}$ \\
\hline 25 & $71.4 \mathrm{~s}$ & $83.1 \mathrm{~s}$ & $83.1 \mathrm{~s}$ & $71.0 \mathrm{~s}$ & $71.9 \mathrm{~s}$ & $71.4 \mathrm{~s}$ & $70.9 \mathrm{~s}$ & $70.9 \mathrm{~s}$ & $71.5 \mathrm{~s}$ & $72.1 \mathrm{~s}$ & $72.0 \mathrm{~s}$ & $59.1 \mathrm{~s}$ \\
\hline 26 & $27.6 \mathrm{q}$ & $23.4 \mathrm{q}$ & $23.4 \mathrm{q}$ & $27.0 \mathrm{q}$ & $27.1 \mathrm{q}$ & $28.4 \mathrm{q}$ & $27.1 \mathrm{q}$ & $27.1 \mathrm{q}$ & $28.4 \mathrm{q}$ & $26.8 \mathrm{q}$ & $26.8 \mathrm{q}$ & $25.2 \mathrm{q}$ \\
\hline 27 & $25.9 \mathrm{q}$ & $21.5 \mathrm{q}$ & $21.5 \mathrm{q}$ & $25.6 \mathrm{q}$ & $25.4 \mathrm{q}$ & $25.2 \mathrm{q}$ & $25.4 \mathrm{q}$ & $25.4 \mathrm{q}$ & $25.1 \mathrm{q}$ & $28.4 \mathrm{q}$ & $28.4 \mathrm{q}$ & $19.8 \mathrm{q}$ \\
\hline 28 & $12.3 \mathrm{q}$ & $11.8 \mathrm{q}$ & $11.8 \mathrm{q}$ & $18.3 \mathrm{q}$ & $19.5 q$ & $27.9 q$ & $18.4 \mathrm{q}$ & $18.2 \mathrm{q}$ & $25.3 q$ & $17.6 \mathrm{q}$ & $21.6 \mathrm{q}$ & $20.0 \mathrm{q}$ \\
\hline 29 & $26.0 \mathrm{q}$ & $25.7 q$ & $25.7 \mathrm{q}$ & $26.1 \mathrm{q}$ & $26.3 q$ & $23.2 \mathrm{q}$ & $22.6 q$ & $21.7 q$ & $25.7 q$ & $26.1 \mathrm{q}$ & $26.1 \mathrm{q}$ & $26.3 q$ \\
\hline 30 & $15.7 q$ & $15.4 \mathrm{q}$ & $15.4 \mathrm{q}$ & $13.6 \mathrm{q}$ & $13.9 q$ & $20.8 q$ & $20.2 q$ & $18.9 q$ & $15.1 \mathrm{q}$ & $14.9 \mathrm{q}$ & $13.6 \mathrm{q}$ & $14.7 \mathrm{q}$ \\
\hline \multicolumn{13}{|l|}{ 3-Ara } \\
\hline $1^{\prime}$ & $105.0 \mathrm{~d}$ & $107.3 d$ & $106.8 d$ & & & & & & & & & $108.1 \mathrm{~d}$ \\
\hline $\mathbf{2}^{\prime}$ & $74.5 d$ & $73.2 \mathrm{~d}$ & $69.7 d$ & & & & & & & & & $73.4 \mathrm{~d}$ \\
\hline $3^{\prime}$ & $73.0 \mathrm{~d}$ & $72.5 \mathrm{~d}$ & $76.9 \mathrm{~d}$ & & & & & & & & & $75.2 \mathrm{~d}$ \\
\hline $4^{\prime}$ & $70.3 d$ & $72.3 d$ & $66.7 \mathrm{~d}$ & & & & & & & & & $70.2 d$ \\
\hline $5^{\prime}$ & $67.6 \mathrm{t}$ & $63.2 \mathrm{t}$ & $66.2 \mathrm{t}$ & & & & & & & & & $66.5 \mathrm{t}$ \\
\hline $15-\mathrm{OCOCH}_{3}$ & & & & & & & & & & & & $170.9 \mathrm{~s}$ \\
\hline $15-\mathrm{OCOCH}_{3}$ & & & & & & & & & & & & $21.4 \mathrm{q}$ \\
\hline 23-OCOCH & & & & & & & & & & & & $171.2 \mathrm{~s}$ \\
\hline $23-\mathrm{OCOC} \mathrm{H}_{3}$ & & & & & & & & & & & & $21.5 \mathrm{q}$ \\
\hline $24-\mathrm{OCOCH}_{3}$ & & & & & & & & & & $171.1 \mathrm{~s}$ & $171.1 \mathrm{~s}$ & \\
\hline $24-\mathrm{OCOC} \mathrm{H}_{3}$ & & & & & & & & & & $21.0 \mathrm{~s}$ & $20.9 s$ & \\
\hline $25-\mathrm{OCOCH}_{3}$ & & $170.2 \mathrm{~s}$ & $170.2 \mathrm{~s}$ & & & & & & & & & \\
\hline $25-\mathrm{OCOC} \mathrm{H}_{3}$ & & $21.2 \mathrm{q}$ & $22.3 \mathrm{q}$ & & & & & & & & & \\
\hline $3^{\prime}-\mathrm{OCOCH}_{3}$ & & & $170.8 \mathrm{~s}$ & & & & & & & & & \\
\hline $3^{\prime}-\mathrm{OCOCH}_{3}$ & & & $21.6 \mathrm{q}$ & & & & & & & & & \\
\hline $4^{\prime}-\mathrm{OCOCH}_{3}$ & & $170.8 \mathrm{~s}$ & & & & & & & & & & \\
\hline $4^{\prime}-\mathrm{OCOCH}_{3}$ & & $22.2 \mathrm{q}$ & & & & & & & & & & \\
\hline $\begin{array}{l}4^{\prime}- \\
\mathrm{OCOCH}=\mathrm{CH}-- \\
\mathrm{CH}_{3}\end{array}$ & $166.3 \mathrm{~s}$ & & & & & & & & & & & \\
\hline $\begin{array}{l}4^{\prime}- \\
\mathrm{OCOCH}=\mathrm{CH}-- \\
\mathrm{CH}_{3}\end{array}$ & $122.3 d$ & & & & & & & & & & & \\
\hline $\begin{array}{l}4^{\prime}- \\
\mathrm{OCOCH}=\underline{\mathrm{CH}}-\mathbf{-} \\
\mathrm{CH}_{3}\end{array}$ & $145.2 d$ & & & & & & & & & & & \\
\hline $\begin{array}{l}4^{\prime}- \\
\mathrm{OCOCH}=\mathrm{CH}- \\
\underline{\mathrm{CH}}_{3}\end{array}$ & $18.2 \mathrm{q}$ & & & & & & & & & & & \\
\hline
\end{tabular}




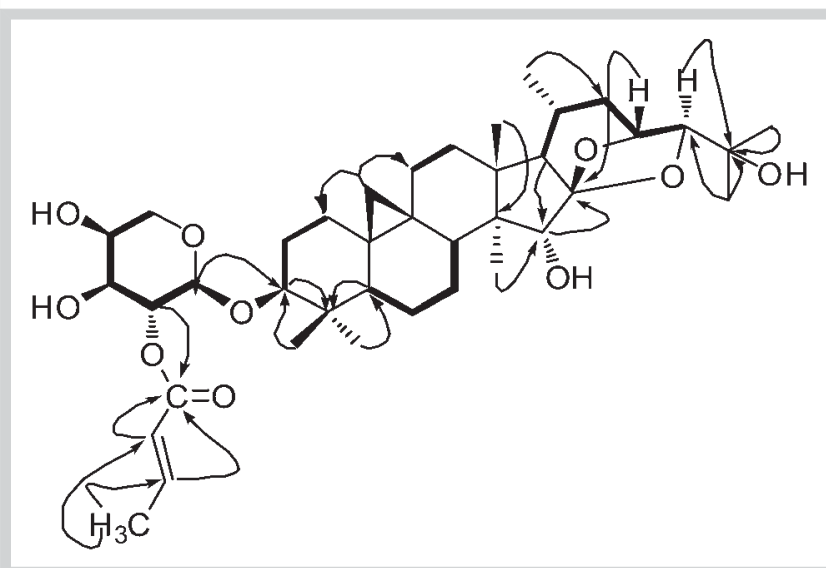

Fig. 2 Major $\mathrm{HMBC}(\rightarrow)$ and ${ }^{1} \mathrm{H}-{ }^{1} \mathrm{H} \operatorname{COSY}(-)$ correlations of compound 1.

\section{Cytotoxicity bioassay}

Five human cancer cell lines, human myeloid leukemia HL-60, hepatocellular carcinoma SMMC-7721, lung cancer A-549, breast cancer MCF-7, and colon cancer SW480, were used in the cytotoxic assay. Cells were cultured in DMEM medium (Hyclone) supplemented with $10 \%$ fetal bovine serum (Hyclone), in $5 \% \mathrm{CO}_{2}$ at $37^{\circ} \mathrm{C}$. The cytotoxicity assay was performed according to the MTT [3-(4,5-dimethylthiazol-2-yl)-2,5-diphenyl tetrazolium bromide] method in 96-well microplates [32,33]. Briefly, $100 \mu \mathrm{L}$ of adherent cells were seeded into each well of 96-well cell culture plates and allowed to adhere for $12 \mathrm{~h}$ before addition of test compounds, while suspended cells were seeded just before drug addition with an initial density of $1 \times 10^{5}$ cells $/ \mathrm{mL}$. Each tumor cell line was exposed to the test compounds (dissolved in DMSO, then diluted by DMEM medium) at concentrations of $0.064,0.32$, $1.6,8$, and $40 \mu \mathrm{M}$ in triplicate for $48 \mathrm{~h}$, with cisplatin (Sigma) and culture solution as positive and negative controls, respectively. After compound treatment, cell viability was detected, and a cell growth curve was graphed. $\mathrm{IC}_{50}$ values were calculated by Reed and Muench's method [34].

\section{Supporting information}

The NMR spectra of compounds 1-12, as well as ESI and HR-TOF-ESI data of 1-12 are available as Supporting Information.

\section{Results and Discussion}

$\nabla$

Compound 1 was obtained as white powder. Its molecular formula $\left(\mathrm{C}_{39} \mathrm{H}_{60} \mathrm{O}_{10}\right)$ was deduced from the analysis of ${ }^{13} \mathrm{C}$ NMR and HR-TOF-ESIMS data $\left\{\mathrm{m} / \mathrm{z}: 711.4065\left[\mathrm{M}+\mathrm{Na}^{+}\right.\right.$(calc. for $\left.\left.\mathrm{C}_{39} \mathrm{H}_{60} \mathrm{O}_{10} \mathrm{Na}, 711.4084\right)\right\}$. The IR spectrum showed absorptions for hydroxy groups at $3452 \mathrm{~cm}^{-1}$, carbonyl groups at $1727 \mathrm{~cm}^{-1}$, and double bonds at $1632 \mathrm{~cm}^{-1}$, respectively. The ${ }^{1} \mathrm{H}-\mathrm{NMR}$ spectrum ( $\odot$ Table 1 ) showed the presence of the characteristic cyclopropane methylene signals at $\delta_{\mathrm{H}} 0.21$ and 0.45 (each $1 \mathrm{H}$, brs), an anomeric proton at $\delta_{\mathrm{H}} 4.79(\mathrm{~d}, J=7.5 \mathrm{~Hz})$, two olefinic protons at $\delta_{\mathrm{H}} 6.05(1 \mathrm{H}, \mathrm{d}, J=15.5 \mathrm{~Hz})$ and $7.09(1 \mathrm{H}, \mathrm{m})$, two secondary methyl signals at $\delta_{\mathrm{H}} 0.84(\mathrm{~d}, J=7.0 \mathrm{~Hz})$ and $1.63(\mathrm{~d}, J=6.5 \mathrm{~Hz})$, and six tertiary methyl groups at $\delta_{\mathrm{H}} 0.95-1.48$. In the ${ }^{13} \mathrm{C}$ and DEPT NMR spectra of 1 ( $\odot$ Table 2 ), the signals ascribable to an $\alpha, \beta$-unsaturated ketone moiety at $\delta_{\mathrm{C}} 166.3,122.3$, and 145.2 were observed. A comparison of the spectroscopic data of 1 with those of cimige-

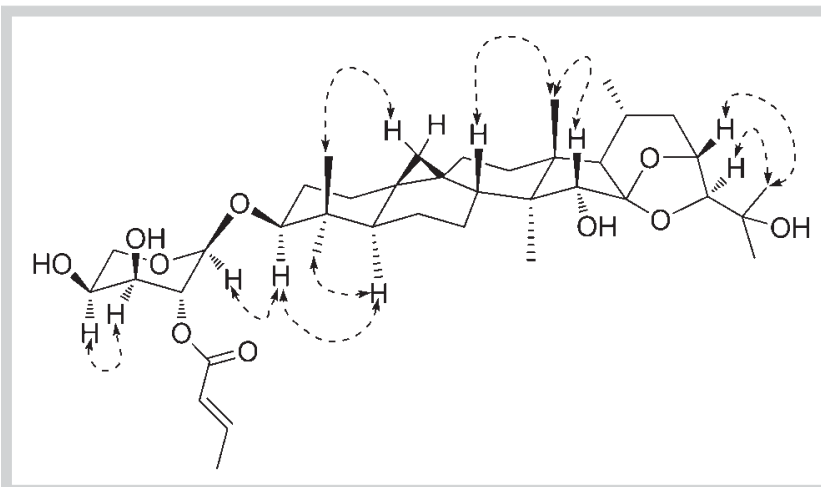

Fig. 3 Key NOESY correlations of compound $\mathbf{1}$.

nol-3-O- $\alpha$-L-arabinopyranoside (27) [15] showed that, structurally, 1 closely resembles $\mathbf{2 7}$, with the main differences of the sugar unit and the presence of another tetra-carbon unit, including the $\alpha, \beta$-unsaturated ketone resonances. In the ${ }^{1} \mathrm{H}-{ }^{1} \mathrm{H}$ COSY spectrum ( $\odot$ Fig. 2), a correlation was observed between the secondary methyl signal at $\delta_{\mathrm{H}} 1.63(\mathrm{~d}, J=6.5 \mathrm{~Hz})$ and the olefinic proton at $\delta_{\mathrm{H}} 7.09(\mathrm{~m})$, which indicated the tetra-carbon unit to be a 2-butenoyl. In addition, the coupling constant $(J=15.6 \mathrm{~Hz})$ of the two olefinic protons at $\delta_{\mathrm{H}} 6.05$ and 7.09 confirmed the E-geometry of a double bond in the 2-butenoyl moiety. In the HMBC spectrum ( Fig. 2), a correlation was observed between the anomeric proton at $\delta_{\mathrm{H}} 4.79\left(\mathrm{H}-1^{\prime}, 1 \mathrm{H}, \mathrm{d}, J=7.5 \mathrm{~Hz}\right)$ and the methine signal at $\delta_{\mathrm{C}}$ 89.2 (C-3), suggesting that a sugar unit was attached at the C-3. The sugar obtained after acid hydrolysis was identified as $\alpha$-Larabinose by comparing its TLC and specific rotation with the standard. In the ${ }^{1} \mathrm{H}$ NMR spectrum, a downfield resonance was observed at $\delta_{\mathrm{H}} 5.99(\mathrm{t}, J=8.5 \mathrm{~Hz})$, which showed correlations with the methine resonance at $\delta_{\mathrm{H}} 4.30\left(\mathrm{H}-3^{\prime}\right)$ and the anomeric proton at $\delta_{\mathrm{H}} 4.79$ in the ${ }^{1} \mathrm{H}-{ }^{1} \mathrm{H}$ COSY spectrum ( $\bullet$ Fig. 2). Furthermore, the HMBC ( $\bullet$ Fig. 2 ) correlation between the carbonyl group $\left(\delta_{\mathrm{C}}\right.$ $166.3)$ and the proton resonance $\left(\delta_{\mathrm{H}} 5.99, \mathrm{t}, J=8.5 \mathrm{~Hz}\right)$ indicated the $(E)-2$-butenoyl unit was attached at C-2'. In the ROESY spectrum ( Fig. 3), H-3 showed a correlation with $\mathrm{H}-5$ suggesting an $\alpha$-orientation of the $\mathrm{H}-3$, while $\mathrm{H}-15$ showed a correlation with Me-18, indicating an $\alpha$-orientation of the hydroxyl group at C15. The configuration of $\mathrm{C}-23$ and $\mathrm{C}-24$ was deduced as $R$ and $S$, respectively, by comparison of the coupling constant of $\mathrm{H}-23$ and $\mathrm{H}-24$ with those of cimigenol-type compounds $[6,22,26]$. Therefore, the structure of 1 was determined as cimigenol-3-O[2'-O-(E)-2-butenoyl]- $\alpha$-L-arabinopyranoside.

Compounds $\mathbf{2}$ and $\mathbf{3}$ were determined to have the same molecular formula $\mathrm{C}_{39} \mathrm{H}_{60} \mathrm{O}_{11}$ by HR-TOF-ESIMS ( $\mathrm{m} / z$ 727.4029 [M + Na] ${ }^{+}$ and $703.4055[\mathrm{M}-\mathrm{H}]$, respectively). The NMR spectroscopic data ( Tables $\mathbf{1}$ and $\mathbf{2}$ ) of $\mathbf{2}$ and $\mathbf{3}$ were similar to those of 25-O-acetylcimigenol-3-O-[2'-O-acetyl]- $\alpha$-L-arabinopyranoside (19), except for the sugar moiety. In the ${ }^{1} \mathrm{H}-\mathrm{NMR}$ spectrum ( $\bullet$ Table $\mathbf{1}$ ) of $\mathbf{2}$, the signal due to $\mathrm{H}-4^{\prime}$ showed a downfield shift from $\delta_{\mathrm{H}} 4.16$ to 5.50. Meanwhile, the signal of $\mathrm{H}-2^{\prime}$ ' was shifted from $\delta_{\mathrm{H}} 5.89$ to $\delta_{\mathrm{H}} 4.38$. In addition, the signal due to $\mathrm{C}-4^{\prime}$ exhibited a downfield shift from $\delta_{\mathrm{C}} 69.8$ to 72.3 in the ${ }^{13} \mathrm{C}$ NMR spectrum (- Table 2 ). The changes of these chemical shifts may be explained by the 0 acetyl group being attached to $\mathrm{C}-4$ of the sugar unit, which was further confirmed by the presence of the HMBC correlation between the $\mathrm{H}-4$ ' signal at $\delta_{\mathrm{H}} 5.50$ and the carbonyl group signal at $\delta_{\mathrm{C}} 170.8$. The sugar obtained after acid hydrolysis was confirmed 
as $\alpha$-L-arabinopyranose by comparing its TLC and specific rotation with the standard. The configurations of C-23 and C-24 are proposed as $R$ and $S$, respectively, by the same way as that of 1 . Thus, the structure of $\mathbf{2}$ was assigned as 25- 0 -acetylcimigenol$3-0$-[4'-O-acetyl]- $\alpha$-L-arabinopyranoside. In the same way, an acetoxy group was determined to be at $\mathrm{C}-3^{\prime}$ for $\mathbf{3}$, which was further confirmed by the presence of the HMBC correlation between the $\mathrm{H}-3^{\prime}$ signal at $\delta_{\mathrm{H}} 5.48$ and the carbonyl group signal at $\delta_{\mathrm{C}}$ 170.8. Therefore, 3 was identified as 25-O-acetylcimigenol-3-O[3'-O-acetyl]- $\alpha$-L-arabinopyranoside.

Compound 4 gave a molecular formula of $\mathrm{C}_{30} \mathrm{H}_{46} \mathrm{O}_{6}$ as established by HR-TOF-ESIMS $\left(\mathrm{m} / \mathrm{z} 525.3192[\mathrm{M}+\mathrm{Na}]^{+}\right)$, which is 16 Da more than those of 7(8)-en-cimigenol (14). The ${ }^{1} \mathrm{H}$ NMR spectrum ( Table 1) displayed downfield cyclopropane methylene signals at $\delta_{\mathrm{H}} 0.73(1 \mathrm{H}, \mathrm{d}, J=4.0 \mathrm{~Hz})$ and 1.19 (overlapped), seven methyl groups at $\delta_{\mathrm{H}} 1.07-1.52$, and an olefinic proton at $\delta_{\mathrm{H}} 6.20(1 \mathrm{H}, \mathrm{d}$, $J=7.0 \mathrm{~Hz}$ ), respectively, suggesting 4 to be a 9,19-cyclolanostane aglycone with a pair of olefinic carbons close to the cyclopropane methylene at C-19 [20]. The NMR data of 4 showed a close resemblance with those of 14 , except that the methylene signal at $\delta_{\mathrm{C}}$ 33.7 ( $\mathrm{C}-12$ ) was absent, showing instead a hydroxymethine at $\delta_{\mathrm{C}}$ 72.5. On the basis of the above observations, it was reasonable to deduce that 4 was a 12-hydroxy derivative of 14 , which was also supported by the $\mathrm{HMBC}$ correlations of $\mathrm{H}-12$ at $\delta_{\mathrm{H}} 4.36$ with $\mathrm{C}-11$ ( $\left.\delta_{\mathrm{C}} 40.3\right), \mathrm{C}-13\left(\delta_{\mathrm{C}} 47.1\right)$, and $\mathrm{CH}_{3}-18\left(\delta_{\mathrm{C}} 13.2\right)$, as well as by the downfield shift of $\mathrm{C}-11$ about $14.9 \mathrm{ppm}$ in the ${ }^{13} \mathrm{C}$ spectrum ( Table 2). Significant ROESY correlations of $\mathrm{H}-12$ with $\mathrm{H}-5$, and $\mathrm{CH}_{3}-28$ suggested a $\beta$-orientation of the substituent at $\mathrm{C}-12$. Therefore, 4 was elucidated as $12 \beta$-hydroxy-7(8)-en-cimigenol. Compound 5 was assigned as $\mathrm{C}_{30} \mathrm{H}_{46} \mathrm{O}_{6}$, as deduced from the HR-TOF-ESIMS $\left(m / z 525.3199[\mathrm{M}+\mathrm{Na}]^{+}\right)$, which is identical to that of compound $\mathbf{4}$. The NMR data of $\mathbf{5}$ were similar to that of 4 with the major difference being that a hydroxyl group was shifted from $\mathrm{C}-12$ to $\mathrm{C}-11$. In the ${ }^{13} \mathrm{C}$ NMR spectrum ( Table 2 ) of 5 , the signal due to $\mathrm{C}-12$ exhibited an upfield shift from $\delta_{\mathrm{C}} 72.5$ to 49.4 , while C-11 showed a downfield shift from $\delta_{C} 40.3$ to 63.5 , further confirming the deduction. The relative configuration of the hydroxyl group at $\mathrm{C}-11$ was proposed as $\beta$-orientated by analyses of the ROESY spectrum. Accordingly, compound $\mathbf{5}$ was characterized as $11 \beta$-hydroxy-7(8)-en-cimigenol.

Compound 6 had the molecular formula $\mathrm{C}_{30} \mathrm{H}_{44} \mathrm{O}_{5}$ from its positive mode HR-TOF-ESIMS (507.3090 [M + Na $]^{+}$). In the ${ }^{13} \mathrm{C}$ and DEPT spectra ( Table 2), 6 exhibited signals very similar to those of 5 except that hydroxy methine signals due to C-3 and C-15 were absent, whereas a carbonyl carbon at $\delta_{\mathrm{C}} 216.1$ and a downfield methylene at $\delta_{\mathrm{C}} 45.8$ were observed. In addition, HMBC correlations of $\mathrm{H}-2, \mathrm{CH}_{3}-29$, and $\mathrm{CH}_{3}-30$ with the carbonyl group at $\delta_{\mathrm{C}} 216.1$ and protons $\left(\delta_{\mathrm{H}} 2.46\right.$ and 2.28 , each $\left.1 \mathrm{H}\right)$ due to the methylene at $\delta_{\mathrm{C}} 45.8$ with $\mathrm{C}-14, \mathrm{C}-16$, and $\mathrm{CH}_{3}-28$ indicated that a carbonyl carbon replaced a hydroxyl group at $\mathrm{C}-3$, and the methine at $\mathrm{C}-15$ was transformed to a methylene in $\mathbf{6}$. Ultimately, $\mathbf{6}$ was elucidated as $11 \beta$-hydroxy-15-deoxycimigenol-7(8)-en-3-one.

The HR-TOF-ESIMS ( $\left.m / z 507.3086[\mathrm{M}+\mathrm{Na}]^{+}\right)$of compound $7 \mathrm{de}-$ termined its molecular formula as $\mathrm{C}_{30} \mathrm{H}_{44} \mathrm{O}_{5}$, which is identical with 24-epi-cimigenol-7(8)-en-3-one (17). The NMR data ( Tables 1 and 2) of 7 resembled those of 17 with major differences at C-22 $\left(\delta_{\mathrm{C}} 38.0\right), \mathrm{C}-23\left(\delta_{\mathrm{C}} 72.1\right), \mathrm{C}-24\left(\delta_{\mathrm{C}} 90.3\right)$, and C-25 $\left(\delta_{\mathrm{C}}\right.$ $70.9)$, which are similar to the key structural differences between cimigenol-type and 24-epi-cimigenol-type triterpenes [21]. By comparing the coupling constant of $\mathrm{H}-24(0 \mathrm{~Hz})$ with those of cimigenol-type compounds $(0 \mathrm{~Hz})$ and 24-epi-cimigenol-type constituents $(4 \mathrm{~Hz})$, the configuration of $\mathrm{C}-24$ of 7 was determined to be $S[6,21,25]$. Thus, 7 was elucidated as cimigenol-7(8)-en-3one.

The molecular formula of compound $\mathbf{8}$ was determined as $\mathrm{C}_{30} \mathrm{H}_{42} \mathrm{O}_{5}$ from the HR-TOF-ESIMS ( $m / z$ 505.2944 [M + Na] $\left.{ }^{+}\right)$. Its NMR data ( Tables 1 and 2) were similar to those of 7 except for the signals of ring A. Unsaturated carbon signals at $\delta_{\mathrm{C}} 152.6$ and 127.2 were observed in $\mathbf{8}$, whereas signals of two methylenes due to $\mathrm{C}-1$ and $\mathrm{C}-2$ were absent. Significant HMBC correlations were observed between the carbonyl C-atom at $\delta_{\mathrm{C}} 203.8$ and the olefinic protons at $\delta_{\mathrm{H}} 6.16$ and 6.71 (each $1 \mathrm{H}, \mathrm{d}, J=10.0 \mathrm{~Hz}$ ). The above evidence suggested that compound $\mathbf{8}$ is transformed from 7 through dehydrogenation between $\mathrm{C}-1$ and $\mathrm{C}-2$. Therefore, compound 8 was characterized as cimigenol-1(2),7(8)-dien-3one.

Compound 9 was assigned a molecular formula of $\mathrm{C}_{30} \mathrm{H}_{46} \mathrm{O}_{4}$ from its HR-TOF-ESIMS $\left(\mathrm{m} / \mathrm{z} 469.3311[\mathrm{M}+\mathrm{H}]^{+}\right)$. In the ${ }^{1} \mathrm{H}$ NMR spectrum, signals for the significant downfield cyclopropane methylene at $\delta_{\mathrm{H}} 3.15$ and $3.24(1 \mathrm{H}$ each, d, $J=14.0 \mathrm{~Hz}$ ), seven methyl groups at $\delta_{\mathrm{H}}$ 0.81-1.52, and three olefinic protons at $\delta_{\mathrm{H}} 5.39$, 5.52, and 5.54 were observed, suggesting 9 is a 9,10-seco-9,19cycloartane triterpene [30]. The ${ }^{13} \mathrm{C}$ NMR and DEPT spectroscopic data of $\mathbf{9}$ were identical with the aglycone resonances of cimicinol (28) [31], except for the upfield shift of the C-3 by $9.7 \mathrm{ppm}$, which could be explained by the absence of a sugar unit at C-3. Therefore, 9 was elucidated as 9,10-seco-1(10),7(8),9(11)-triencimigenol.

The molecular formula of compound $\mathbf{1 0}$ was established as $\mathrm{C}_{32} \mathrm{H}_{50} \mathrm{O}_{6}$ on the basis of HR-TOF-ESIMS $\left(\mathrm{m} / z 553.3494[\mathrm{M}+\mathrm{Na}]^{+}\right)$. In the IR spectrum, absorption bands at 3473 and $1733 \mathrm{~cm}^{-1}$ for hydroxyl and carbonyl groups were observed. In the ${ }^{13} \mathrm{C}$ and DEPT NMR spectra, the signals ascribable to the methylene carbon of the cyclopropane ring at $\delta_{\mathrm{C}} 31.3$ (C-19), four oxygen-bearing methine carbons at $\delta_{\mathrm{C}} 84.3$ (C-16), 79.8 (C-24), 79.1 (C-23), and 77.9 (C-3), as well as two carbonyl carbons at $\delta_{\mathrm{C}} 213.9$ (C$15)$ and 171.1 ( $C_{24}$-acetoxy) were observed, suggesting that 10 was a highly oxygenated 9,19-cycloartane triterpene aglycon with an 0 -acetyl group. By comparison of NMR spectroscopic data, $\mathbf{1 0}$ was determined to be the aglycon of 24- 0 -acetylisodahurinol-3- 0 - $\alpha$-L-arabinopyranoside (29) [7]. The configuration of C24 was deduced as $S$ by comparison of the coupling constants of $\mathrm{H}-24(1.6 \mathrm{~Hz})$ with those of dahurinyl diacetate $(9 \mathrm{~Hz})$ and isodahurinyl diacetate $(2 \mathrm{~Hz})$ [29]. Accordingly, compound 10 was characterized as 24-O-acetylisodahurinol.

Compound 11 gave a molecular formula of $\mathrm{C}_{32} \mathrm{H}_{48} \mathrm{O}_{6}$ by HR-TOFESIMS at $m / z 551.3346[\mathrm{M}+\mathrm{Na}]^{+}$. The NMR spectroscopic data ( Tables 1 and 2) of $\mathbf{1 1}$ resembled those of $\mathbf{1 0}$ except for major differences at C-7 $\left(\delta_{\mathrm{C}} 115.0\right)$ and C-8 $\left(\delta_{\mathrm{C}} 141.9\right)$ due to dehydrogenation at these positions. This deduction was confirmed by the correlations of $\delta_{\mathrm{H}} 6.36(\mathrm{H}-7)$ with $\delta_{\mathrm{H}} 1.56$ and $1.93(\mathrm{H}-6)$ in the ${ }^{1} \mathrm{H}-{ }^{1} \mathrm{H}$ COSY spectrum. Accordingly, compound 11 was characterized as 24-O-acetyl-7(8)-en-isodahurinol.

Compound 12 exhibited the molecular formula $\mathrm{C}_{39} \mathrm{H}_{58} \mathrm{O}_{11}$, as established by HR-TOF-ESIMS at $m / z 725.3895[\mathrm{M}+\mathrm{Na}]^{+}$. The molecular weight of 12 is 42 Da more than that of 23-0-acetyl-7(8)en-shengmanol-3-O- $\alpha$-L-arabinopyranoside (30) [27], which may be due to an acetyl group. When its spectroscopic data ( Tables 1 and 2) were compared with those of $\mathbf{3 0}$ [27], an additional $O$-acetyl group was assigned to $\mathrm{C}-15$ on the basis of the upfield shift of the carbonyl carbon (C-16) from $\delta_{\mathrm{C}} 220.3$ to 214.2, the downfield shift of $\mathrm{H}-15$ from $\delta_{\mathrm{H}} 4.56$ to 5.92 , as well as the $\mathrm{HMBC}$ correlation of $\mathrm{H}-15$ and the carbonyl group signal at $\delta_{\mathrm{C}}$ 170.9. Significant ROESY correlations of $\mathrm{H}-15$ with $\mathrm{CH}_{3}-18$ sug- 
Table 3 Cytotoxicity ${ }^{a}\left(\mathrm{IC}_{50}, \mu \mathrm{M} \pm \mathrm{SD}\right)$ of compounds isolated from the roots of $C$. dahurica.

\begin{tabular}{|c|c|c|c|c|c|}
\hline Compounds & HL-60 & SMMC-7721 & A-549 & MCF-7 & SW480 \\
\hline 1 & $4.2 \pm 0.4$ & $13.4 \pm 1.1$ & $13.8 \pm 0.8$ & $14.2 \pm 0.7$ & $11.8 \pm 1.3$ \\
\hline 2 & $8.1 \pm 0.5$ & $12.2 \pm 1.2$ & $14.5 \pm 0.7$ & $12.7 \pm 1.4$ & $13.1 \pm 0.9$ \\
\hline 3 & $5.8 \pm 0.2$ & $8.7 \pm 0.3$ & $10.3 \pm 1.1$ & $12.6 \pm 0.5$ & $11.3 \pm 1.2$ \\
\hline 4 & $>40$ & $>40$ & $>40$ & $>40$ & $>40$ \\
\hline 5 & $>40$ & $>40$ & $>40$ & $>40$ & $>40$ \\
\hline 6 & $18.9 \pm 1.6$ & $19.5 \pm 0.7$ & $21.1 \pm 1.8$ & $23.7 \pm 1.5$ & $20.3 \pm 1.7$ \\
\hline 7 & $21.1 \pm 1.5$ & $18.3 \pm 1.7$ & $20.5 \pm 1.0$ & $19.2 \pm 0.9$ & $>40$ \\
\hline 8 & $21.9 \pm 2.2$ & $17.4 \pm 1.8$ & $19.5 \pm 0.8$ & $17.5 \pm 1.3$ & $22.5 \pm 1.2$ \\
\hline 9 & $>40$ & $>40$ & $>40$ & $>40$ & $>40$ \\
\hline 10 & $>40$ & $>40$ & $>40$ & $>40$ & $>40$ \\
\hline 11 & $>40$ & $>40$ & $>40$ & $>40$ & $>40$ \\
\hline 12 & $>40$ & $>40$ & $>40$ & $>40$ & $>40$ \\
\hline 13 & $>40$ & $>40$ & $>40$ & $>40$ & $>40$ \\
\hline 14 & $>40$ & $>40$ & $>40$ & $>40$ & $>40$ \\
\hline 15 & $10.5 \pm 0.9$ & $22.5 \pm 1.3$ & $19.1 \pm 2.1$ & $20.6 \pm 1.4$ & $13.8 \pm 0.8$ \\
\hline 16 & $20.2 \pm 0.7$ & $22.9 \pm 1.5$ & $20.4 \pm 1.7$ & $21.1 \pm 1.2$ & $21.3 \pm 1.4$ \\
\hline 17 & $>0$ & $>40$ & $>40$ & $>40$ & $>0$ \\
\hline 18 & $20.2 \pm 0.8$ & $22.9 \pm 1.7$ & $20.4 \pm 1.3$ & $15.1 \pm 1.2$ & $>40$ \\
\hline 19 & $5.8 \pm 0.7$ & $8.7 \pm 0.8$ & $10.3 \pm 2.1$ & $12.6 \pm 1.7$ & $11.3 \pm 0.7$ \\
\hline 20 & $8.1 \pm 0.6$ & $12.2 \pm 1.4$ & $14.5 \pm 1.2$ & $12.7 \pm 2.3$ & $13.1 \pm 1.5$ \\
\hline 21 & $>40$ & $>40$ & $>40$ & $>40$ & $>40$ \\
\hline 22 & $>40$ & $>40$ & $>40$ & $>40$ & $>40$ \\
\hline 23 & $>40$ & $>40$ & $>40$ & $>40$ & $>40$ \\
\hline 24 & $>40$ & $>40$ & $>40$ & $>40$ & $>40$ \\
\hline 25 & $>40$ & $>40$ & $>40$ & $>40$ & $>40$ \\
\hline 26 & $>40$ & $>40$ & $>40$ & $>40$ & $>40$ \\
\hline Cisplatin & $0.52 \pm 0.0$ & $13.4 \pm 0.6$ & $12.4 \pm 0.7$ & $15.0 \pm 1.4$ & $14.4 \pm 1.1$ \\
\hline
\end{tabular}

${ }^{a}$ Cytotoxicity is the average $(n=3)$ of calculated $\mathrm{IC}_{50} \mathrm{~S}$; the purity of compounds $\mathbf{1 - 2 6}$ is greater than $95 \%$ and of cisplatin greater than $99 \%$

gested an $\alpha$-orientation of the substituent at C-15. The configurations of $\mathrm{C}-23$ and $\mathrm{C}-24$ were considered to be $R$ and $S$, respectively, by comparing coupling constants of $\mathrm{H}-23$ and $\mathrm{H}-24$ with those of known 9,19-cyclolanostane triterpene glycosides [25]. In the ${ }^{13} \mathrm{C}$ NMR spectrum ( Table 2 ), compound 12 showed resonances corresponding to an $\alpha$-L-arabinose moiety at $\delta_{\mathrm{C}} 108.1$ (d), 73.4 (d), $75.2(d), 70.2(d)$, and $66.5(t)[8,29]$, which was further confirmed by comparing its TLC and specific rotation with the standard after hydrolysis. Ultimately, 12 was elucidated as 15,23-Odiacetyl-7(8)-en-shengmanol-3-O- $\alpha$-L-arabinopyranoside.

All isolated compounds were screened for their in vitro antitumor activities. As summarized in $\$$ Table 3, the new compounds 1-3 and the known compounds 19 and 20 showed broad-spectrum and moderate cytotoxicities against human HL-60, SMMC7721, A549, MCF-7, and SW480 cell lines, with $\mathrm{IC}_{50}$ values ranging from 4.2 to $14.5 \mu \mathrm{M}$. In addition, the new compounds 6-8 and the known compounds 15, 16, and 18 exhibited broad-spectrum and week cytotoxicities, having $\mathrm{IC}_{50}$ values around $20 \mu \mathrm{M}$. Based on the above results, we suggest that the roots of $C$. dahurica may be another potential resource for promising antitumor agents.

In the present study, structural and bioactive properties of five cimigenol-type glycosides $(\mathbf{1}-\mathbf{3}, \mathbf{1 9}, \mathbf{2 0})$ are completely in accordance with the SAR we proposed before. Thus, the SAR proposed in our previous studies may be used for the design of more potent lead compounds. Furthermore, six cimigenol-type aglycons (6$8,15,16,18$ ) exhibited broad-spectrum and week cytotoxicities. The main structural characters of these compounds are: (1) the configurations of C-23 and C-24 are $R$ and S, respectively; (2) carbonyl and acetoxy groups instead of a hydroxyl group at C-3 or C25. Previously, we reported that cimigenol-type aglycone actrin- 3-one has potent and moderate activities against human HepG-2 and HT 29 cell lines, respectively. Meanwhile, 25-O-acetylcimigenol exhibited moderate activity against the human HepG-2 cell line [7]. Based on the analyses of these data, we may propose that for cimigenol-type aglycones, hydrophobic groups, such as carbonyl and acetoxy, instead of a hydroxyl group at C-3 or C-25 are essential for cytotoxicity.

\section{Acknowledgments}

$\nabla$

This work was financially supported by the National Natural Science Foundation of China (No. U1132604), Knowledge Innovation Program of the CAS (Grant No. KZCX2-XB2-15-03, KSCX2EW-R-15), as well as Top Talents Program of Yunnan province (2009C1120), and the Foundation of State Key Laboratory of Phytochemistry and Plant Resources in West China (P2010-ZZ14).

\section{Conflict of Interest}

All authors declare that there are no conflicts of interest. The isolation and structural elucidations were accomplished by Yin Nian, Hai-Yan Wang, Lin Zhou, and Ming-Hua Qiu, and the cell culture and cytotoxicity assay were performed by Jia Su and Yan Li. All the authors knew about this manuscript and had no objection to submitting it. 


\section{References}

1 Pan L, Chai HY, Kinghorn AD. The continuing search for antitumor agents from higher plants. Phytochem Lett 2010; 3: 1-8

2 Newman DJ, Cragg GM. Natural products as sources of new drugs over the 30 years from 1981 to 2010. J Nat Prod 2012; 75: 311-335

3 Hsiao WLW, Liu L. The role of traditional Chinese herbal medicines in cancer therapy from TCM theory to mechanistic insights. Planta Med 2010; 76: 1118-1131

4 Chinese Pharmacopoeia Commission. The Pharmacopoeia of Chinese People's Republic. 2010 edition. Beijing: The Chemical Industry Publishing House; 2010: 68-69

5 Sun LR, Qing C, Zhang YL, Ji SY, Li ZR, Pei SJ, Qiu MH, Gross ML, Qiu SX. Cimicifoetisides A and B, two cytotoxic cycloartane triterpenoid glycosides from the rhizomes of Cimicifuga foetida, inhibit proliferation of cancer cells. Beilstein J Org Chem 2007; 3: 1-6

6 Lu L, Chen JC, Song HJ, Li Y, Nian Y, Qiu MH. Five new triterpene bisglycosides with acyclic side chains from the rhizomes of Cimicifuga foetida $\mathrm{L}$. Chem Pharm Bull 2009; 58: 729-733

7 Nian Y, Zhang YL, Chen JC, Lu L, Qing C, Qiu MH. Cytotoxic chemical constituents from the roots of Cimicifuga foetida. J Nat Prod 2010; 73: 9398

8 Nian Y, Zhang XM, Li Y, Wang YY, Chen JC, Lu L, Zhou L, Qiu MH. Cycloartane triterpenoids from the aerial parts of Cimicifuga foetida Linnaeus. Phytochemistry 2011; 72: 1473-1481

9 Sakurai N, Inoue T, Nagai M. Studies on the Chinese crude drug "Shoma." II. Triterpenes of Cimicifuga dahurica MAXIM. Yakugaku Zasshi 1972; 92: 724-728

10 Kimura O, Sakurai N, Inoue T. Studies on the Chinese crude drug "Shoma." VII. Isolation and determination of genuine natural products, acetyl shengmanol xyloside, 24-0-acetylhydroshengmanol xyloside, and shengmanol xyloside, in Cimicifuga dahurica and the other Cimicifuga plants. Yakugaku Zasshi 1983; 103: 293-299

$11 \mathrm{Li}$ CJ, Chen DH, Xiao PG. Studies on the chemical constituents from the aerial parts of Cimicifuga dahurica. Acta Pharm Sin 1993; 28: 777-781

12 Li CJ, Chen DH, Xiao PG. Chemical constituents of traditional Chinese drug "sheng-ma" (Cimicifuga dahurica). II. chemical structure of cimicifugamide. Acta Chim Sin 1994; 52: 296-300

13 Li CJ, Chen DH, Xiao PG. Chemical constituents of traditional Chinese drug "sheng-ma" (Cimicifuga dahurica) III structures of cimiside $C$ and cimiside D. Acta Chim Sin 1994; 52: 722-726

14 Zhang QW, Ye WC, Hsiao W, Zhao SX, Che CT. Cycloartane glycosides from Cimicifuga dahurica. Chem Pharm Bull 2001; 49: 1468-1470

15 Ye WC, Zhang JW, Che CT, Ye T, Zhao SX. Cycloartane glycosides from Cimicifuga dahurica. Planta Med 1999; 65: 770-772

16 Liu Y, Chen DH, Si JY, Tu GZ, An DG. Two new cyclolanostanol xylosides from the aerial parts of Cimicifuga dahurica. J Nat Prod 2002; 65: 14861488

17 Tian Z, Yang MS, Huang F, Li KG, Si JY, Shi L, Chen SB, Xiao PG. Cytotoxicity of three cycloartane triterpenoids from Cimicifuga dahurica. Cancer Lett 2005; 226: 65-75

18 Tian Z, Si JY, Chang Q, Zhou L, Chen SL, Xiao PG, Wu E. Antitumor activity and mechanisms of action of total glycosides from aerial part of Cimici- fuga dahurica targeted against hepatoma. BMC Cancer 2007; 7: 237246

19 Einbonda LS, Ye WC, Hec K, Wu HL, Cruz E, Rollerc M, Kronenberg M. Growth inhibitory activity of extracts and compounds from Cimicifuga species on human breast cancer cells. Phytomedicine 2007; 15: 504511

20 Sun LR, Yan J, Pei SJ, Qiu MH. A new cycloartane triterpenoid from the rhizome of Cimicifuga foetida collected in Dali. Acta Bot Yunn 2005; 27: 331-336

21 Li JX, Kadota S, Hattori M, Yoshimachi S, Shiro M, Oogami N, Mizuno H, Namba T. Isolation and characterization of ten new cycloartenol triterpenes from Cimicifuga heracleifolia Komarov. Chem Pharm Bull 1993; 41: 832-841

22 Gao JC, Zhang JC, Lu ZJ, Zhu GY, Yang MS, Xiao PG. Chemical constituents of Actaea asiatica Hara and their anti-osteoporosis activities. Biochem Syst Ecol 2006; 34: 710-713

23 Kusano A, Takahira M, Shibano M, Miyase T, Okuyama T, Kusano G. Studies on the constituents of Cimicifuga species. XXII. Structure of two new cyclolanostanol xylosides, cimiacerosides A and B. Heterocycles 1998; 48: 1003-1013

24 Zhou L, Yang JS, Tu GZ, Zou JH. Cyclolanostane triterpene glycosides from Souliea vaginata. Chem Pharm Bull 2006; 54: 823-826

25 Chen SN, Fabricant DS, Lu ZZ, Fong HHS, Farnsworth NR. Cimiracemosides I-P, new 9,19-cyclolanostane triterpene glycosides from Cimicifuga racemosa. J Nat Prod 2002; 65: 1391-1397

26 Kusano A, Takahira M, Shibano M, In Y, Ishida T, Miyase T, Kusano G. Studies on the constituents of Cimicifuga species. XX. Absolute stereostructures of cimicifugoside and actein from Cimicifuga simplex WORMSK. Chem Pharm Bull 1998; 46: 467-472

27 Kusano A, Shibano M, Kusano G, Miyase T. Studies on the constituents of Cimicifuga species. XIX. Eight new glycosides from Cimicifuga simplex WORMSK. Chem Pharm Bull 1996; 44: 2078-2085

28 Kusano A, Shibano M, Kitagawa S, Kusano G. Studies on the constituents of Cimicifuga species. XV. Two new diglycosides from the aerial parts of Cimicifuga simplex WORMSK. Chem Pharm Bull 1994; 42: 1940-1943

29 Shao $Y$, Harris A, Wang $M$, Zhang $H$, Cordell GA, Bowman M, Lemmo E. Triterpene glycosides from Cimicifuga racemosa. J Nat Prod 2000; 63: 905-910

30 Ali Z, Khan SI, Fronczek FR, Khan IA. 9,10-seco-9,19-cyclolanostane arabinosides from the roots of Actaea podocarpa. Phytochemistry 2007; 68: 373-382

31 Kadota S, Li JX, Tanaka K, Namba T. Constituents of Cimicifugae rhizoma II. Isolation and structures of new cycloartenol triterpenoids and related compounds from Cimicifuga foetida L. Tetrahedron 1995; 51: 1143-1166

32 Mosmman T. Rapid colorimetric assay for cellular growth and survival: application to proliferation and cytotoxicity assays. Immunol Methods 1983; 65: 55-63

33 Alley MC, Scudiero DA, Monks A. Feasibility of drug screening with panels of human tumor cell lines using a microculture tetrazolium assay. Cancer Res 1988; 48: 589-601

34 Reed $L J$, Muench $H$. A simple method of estimating fifty percent endpoints. Am J Hyg 1938; 27: 493-497 